\title{
Planck pre-launch status: Design and description of the Low Frequency Instrument
}

\author{
M. Bersanelli ${ }^{1,2}$, N. Mandolesi ${ }^{3}$, R. C. Butler ${ }^{3}$, A. Mennella ${ }^{1,2}$, F. Villa ${ }^{3}$, B. Aja ${ }^{4}$, E. Artal ${ }^{4}$, E. Artina ${ }^{5}$, \\ C. Baccigalupi ${ }^{6,15}$, M. Balasini ${ }^{5}$, G. Baldan ${ }^{5}$, A. Banday ${ }^{7,32}$, P. Bastia ${ }^{5}$, P. Battaglia ${ }^{5}$, T. Bernardino ${ }^{8}$, E. Blackhurst ${ }^{9}$, \\ L. Boschini ${ }^{5}$, C. Burigana ${ }^{3}$, G. Cafagna ${ }^{5}$, B. Cappellini ${ }^{1,2}$, F. Cavaliere ${ }^{1}$, F. Colombo $^{5}$, G. Crone ${ }^{10}$, F. Cuttaia ${ }^{3}$, \\ O. D'Arcangelo ${ }^{11}$, L. Danese ${ }^{6}$, R. D. Davies ${ }^{9}$, R. J. Davis ${ }^{9}$, L. De Angelis ${ }^{12}$, G. C. De Gasperis ${ }^{13}$, L. De La Fuente ${ }^{4}$, \\ A. De Rosa ${ }^{3}$, G. De Zotti ${ }^{14}$, M. C. Falvella ${ }^{12}$, F. Ferrari ${ }^{5}$, R. Ferretti ${ }^{5}$, L. Figini ${ }^{11}$, S. Fogliani ${ }^{15}$, C. Franceschet ${ }^{1}$, \\ E. Franceschi ${ }^{3}$, T. Gaier ${ }^{16}$, S. Garavaglia' ${ }^{11}$, F. Gomez ${ }^{17}$, K. Gorski ${ }^{16}$, A. Gregorio ${ }^{18}$, P. Guzzi ${ }^{5}$, J. M. Herreros ${ }^{17}$,
} S. R. Hildebrandt ${ }^{17}$, R. Hoyland ${ }^{17}$, N. Hughes ${ }^{19}$, M. Janssen ${ }^{16}$, P. Jukkala ${ }^{19}$, D. Kettle ${ }^{9}$, V. H. Kilpiä ${ }^{19}$, M. Laaninen ${ }^{20}$, P. M. Lapolla ${ }^{5}$, C. R. Lawrence ${ }^{16}$, D. Lawson ${ }^{9}$, J. P. Leahy ${ }^{9}$, R. Leonardi ${ }^{21}$, P. Leutenegger ${ }^{5}$, S. Levin ${ }^{16}$, P. B. Lilje ${ }^{22}$, S. R. Lowe ${ }^{9}$, P. M. Lubin ${ }^{21}$, D. Maino ${ }^{1}$, M. Malaspina ${ }^{3}$, M. Maris ${ }^{15}$, J. Marti-Canales ${ }^{10}$, E. Martinez-Gonzalez ${ }^{8}$, A. Mediavilla ${ }^{4}$, P. Meinhold ${ }^{21}$, M. Miccolis ${ }^{5}$, G. Morgante ${ }^{3}$, P. Natoli ${ }^{13}$, R. Nesti ${ }^{23}$, L. Pagan ${ }^{5}$, C. Paine ${ }^{16}$, B. Partridge ${ }^{24}$, J. P. Pascual ${ }^{4}$, F. Pasian ${ }^{15}$, D. Pearson ${ }^{16}$, M. Pecora ${ }^{5}$, F. Perrotta ${ }^{15,6}$, P. Platania ${ }^{11}$, M. Pospieszalski ${ }^{25}$, T. Poutanen ${ }^{26,27,28}$, M. Prina ${ }^{16}$, R. Rebolo ${ }^{17}$, N. Roddis ${ }^{9}$, J. A. Rubiño-Martin ${ }^{17}$, M. J. Salmon ${ }^{8}$, M. Sandri ${ }^{3}$, M. Seiffert ${ }^{16}$, R. Silvestri ${ }^{5}$, A. Simonetto ${ }^{11}$, P. Sjoman ${ }^{19}$, G. F. Smoot ${ }^{29}$, C. Sozzi ${ }^{11}$, L. Stringhetti ${ }^{3}$, E. Taddei ${ }^{5}$,

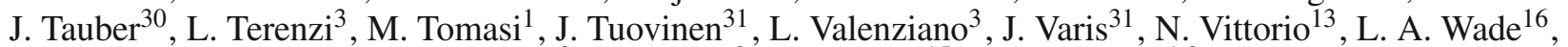
A. Wilkinson ${ }^{9}$, F. Winder ${ }^{9}$, A. Zacchei ${ }^{15}$, and A. Zonca ${ }^{1,2}$

(Affiliations can be found after the references)

Received 8 July 2009 / Accepted 15 December 2009

\section{ABSTRACT}

In this paper we present the Low Frequency Instrument (LFI), designed and developed as part of the Planck space mission, the ESA programme dedicated to precision imaging of the cosmic microwave background (CMB). Planck-LFI will observe the full sky in intensity and polarisation in three frequency bands centred at 30,44 and $70 \mathrm{GHz}$, while higher frequencies $(100-850 \mathrm{GHz})$ will be covered by the HFI instrument. The LFI is an array of microwave radiometers based on state-of-the-art indium phosphide cryogenic HEMT amplifiers implemented in a differential system using blackbody loads as reference signals. The front end is cooled to $20 \mathrm{~K}$ for optimal sensitivity and the reference loads are cooled to $4 \mathrm{~K}$ to minimise low-frequency noise. We provide an overview of the LFI, discuss the leading scientific requirements, and describe the design solutions adopted for the various hardware subsystems. The main drivers of the radiometric, optical, and thermal design are discussed, including the stringent requirements on sensitivity, stability, and rejection of systematic effects. Further details on the key instrument units and the results of ground calibration are provided in a set of companion papers.

Key words. cosmic microwave background - cosmology: observations - space vehicles: instruments

\section{Introduction}

Observations of the cosmic microwave background (CMB) have played a central role in the enormous progress of cosmology in the past few decades. Technological developments in both coherent radio receivers and bolometric detectors have supported an uninterrupted chain of successful experiments, from the initial discovery (Penzias \& Wilson 1965) up to the present generation of precision measurements. Following $\mathrm{COBE}^{1}$ and WMAP $^{2}$, the Planck ${ }^{3}$ satellite, launched on 14 May 2009, is the next-generation space mission dedicated to $\mathrm{CMB}$ observations.

\footnotetext{
1 http://lambda.gsfc.nasa.gov/product/cobe/

2 http://map.gsfc.nasa.gov/

3 Planck (http://Www.esa.int/Planck) is a project of the European Space Agency - ESA - with instruments provided by two scientific Consortia funded by ESA member states (in particular the lead countries: France and Italy) with contributions from NASA (USA), and
}

The Planck instruments are designed to extract all the cosmological information encoded in the CMB temperature anisotropies with an accuracy set by cosmic variance and astrophysical confusion limits, and to push polarisation measurements well beyond previously reached results. Planck will image the sky in nine frequency bands across the CMB blackbody peak, leading to a full-sky map of the CMB temperature fluctuations with signal-to-noise $>10$ and angular resolution $<10^{\prime}$. The Planck instruments and observing strategy were devised to reach an unprecedented combination of angular resolution $\left(5^{\prime}\right.$ to $\left.30^{\prime}\right)$, sky coverage $(100 \%)$, spectral coverage $(27-900 \mathrm{GHz})$, sensitivity $\left(\Delta T / T \sim 2 \times 10^{-6}\right)$, calibration accuracy $(\sim 0.5 \%)$, and rejection of systematic effects $(\sim 1 \mu \mathrm{K}$ per pixel) (Tauber et al. 2010a).

telescope reflectors provided in a collaboration between ESA and a scientific Consortium led and funded by Denmark. 
In addition, all Planck bands between 30 and $350 \mathrm{GHz}$ are sensitive to linear polarisation.

The imaging power of Planck is sized to extract the temperature power spectrum with high precision over the entire angular range dominated by primordial fluctuations. This will lead to accurate estimates of cosmological parameters that describe the geometry, dynamics, and matter-energy content of the universe. The Planck polarisation measurements are expected to deliver complementary information on cosmological parameters and to provide a unique probe of the thermal history of the universe in the early phase of structure formation. Planck will also test the inflationary paradigm with unprecedented sensitivity through studies of non-Gaussianity and of B-mode polarisation as a signature of primordial gravitational waves (Planck Collaboration 2005).

The wide frequency range of Planck is required primarily to ensure accurate discrimination of foreground emissions from the cosmological signal. However, the nine Planck maps will also represent a rich data set for galactic and extragalactic astrophysics. Up to now, no single technology can reach the required performances in the entire Planck frequency range. For this reason two complementary instruments are integrated at the Planck focal plane exploiting state-of-the-art radiometric and bolometric detectors in their best windows of operation. The Low Frequency Instrument (LFI), described in this paper, covers the $27-77 \mathrm{GHz}$ range with a radiometer array cooled to $20 \mathrm{~K}$. The High Frequency Instrument (HFI) will observe in six bands in the 90-900 GHz range with a bolometer array cooled to $0.1 \mathrm{~K}$ (Lamarre et al. 2010). The two instruments share the focal plane of a single telescope, a shielded off-axis dual reflector Gregorian system with $1.5 \times 1.9 \mathrm{~m}$ primary aperture (Tauber et al. 2010b).

The design of the Planck satellite and mission plan is largely driven by the extreme thermal requirements imposed by the instruments. The cold payload enclosure $(<50 \mathrm{~K}$ passive cooling) needs to be thermally decoupled from the warm $(\sim 300 \mathrm{~K})$ service module while preserving high thermal stability. The optical design, orbit, and scanning strategy are optimised to obtain the required effective angular resolution, rejection of stray light, and environmental stability. Planck has been injected into a Lissajous orbit around the Sun-Earth L2 point, at 1.5 million $\mathrm{km}$ from Earth. The scanning strategy assumes, to first order, the spacecraft is spinning at $1 \mathrm{rpm}$ with the spin axis aligned at $0^{\circ}$ solar aspect angle. The typical angle between the detectors' line of sight and the spin axis is $\sim 85^{\circ}$. It will be possible to redirect the spin axis within a cone of $10^{\circ}$ around the spacecraft-sun axis. The baseline mission allows for 15 months of routine scientific operations in L2, a period in which the entire sky can be imaged twice by all detectors. However, in anticipation of a possible extension of the mission, spacecraft and instrument consumables allow an extension by a factor of two ${ }^{4}$.

In this paper we present the design of the Planck-LFI and discuss its driving scientific requirements. We give an overview of the main subsystems, particularly those that are critical for scientific performance, while referring to a set of companion papers for more details. The LFI programme as a whole, including data processing and programming issues, is described in Mandolesi et al. (2010); the calibration plan and ground calibration results are discussed by Mennella et al. (2010) and Villa et al. (2010). The LFI optical design is presented in Sandri et al. (2010), while the expected polarisation performance is discussed in Leahy et al. (2010).

\footnotetext{
4 Currently, the nominal mission lifetime has been extended to 24 months.
}

In Sect. 2.1 we discuss the main scientific requirements of LFI. We start from top-level guidelines such as frequency range, angular resolution, and sensitivity, and then move to more detailed requirements that were derived for the chosen design by assuming a moderate level of extrapolation of the technology available at the time of the design completion. In Sect. 3 we provide an overall description of LFI instrument configuration and discuss in detail the LFI differential radiometers and associated components. Section 4 is a description of the instrument system and subsystems, including optical, radiometric, and electronic units, while Sects. 4 to 7 describe the thermal, electrical, and optical interfaces.

\section{Scientific requirements}

In this section we discuss the main scientific requirements for the LFI. Here, and throughout this paper, we discuss instrument specifications, while measured on-ground performance are discussed in Mennella et al. (2010) and Villa et al. (2010). Measured values are generally in line with the design specifications, although noise levels are somewhat higher, particularly at $44 \mathrm{GHz}$. On the other hand, angular resolution at $70 \mathrm{GHz}$ and stability at all frequencies surpass the requirement values. In the following sections we describe the design solutions implemented to meet such requirements.

\subsection{Frequency range}

The minimum of the combined diffuse emission of foregrounds relative to the CMB spectrum occurs at $\lambda \sim 4 \mathrm{~mm}$, i.e., roughly at the turning point between optimal performances of radiometric coherent receivers and bolometric detectors. Simulations carried out in the early design phases of Planck (Bersanelli et al. 1996a) have shown that a set of four logarithmically spaced bands in the 30-100 GHz range would provide good spectral leverage to disentangle low-frequency components, while covering the window of minimum foregrounds for optimal CMB science. The LFI is designed to cover the frequency range below the peak of the CMB spectrum using an array of differential radiometers (Bersanelli et al. 1996b; Mandolesi et al. 2000b). The initial Planck-LFI configuration (Bersanelli \& Mandolesi 2000) included four bands centred at $30,44,70$, and $100 \mathrm{GHz}$, with the $100 \mathrm{GHz}$ channel covered by both LFI and HFI for scientific redundancy and systematics crosschecks. Budget and managerial difficulties, however, led to descoping of the LFI $100 \mathrm{GHz}$ channel, which is now covered by HFI only. Nonetheless, the three LFI bands centred at 30,44 , and $70 \mathrm{GHz}$ in combination with the six HFI bands provide Planck with a uniquely broad spectral coverage for robust separation of non-cosmological components. In addition, the LFI $70 \mathrm{GHz}$ channel offers the cleanest view of the CMB for both temperature and polarisation anisotropy.

\subsection{Angular resolution and sensitivity}

After neglecting astrophysical foregrounds, calibration errors, and systematic effects, and after taking cosmic variance into account, the uncertainty in the parent distribution of the CMB power spectrum $C_{\ell}$ is given by (Knox 1995):

$$
\frac{\delta C_{\ell}}{C_{\ell}} \simeq f_{\mathrm{sky}}^{-1 / 2} \sqrt{\frac{2}{2 \ell+1}}\left[1+\frac{A \sigma_{\mathrm{pix}}^{2}}{N_{\mathrm{pix}} C_{\ell} W_{\ell}^{2}}\right],
$$

where $\ell$ is the multipole index, $f_{\text {sky }}$ the fraction of CMB sky observed, $A$ the surveyed area, $N_{\text {pix }}$ the number of pixels, $\sigma_{\text {pix }}$ the 
average noise per pixel at the end of the mission, and $W_{\ell}$ the window function, which for LFI can be approximated by $W_{\ell}^{2}=\exp \left[-\ell(\ell+1) \sigma_{B}^{2}\right]$ with $\sigma_{B}=\theta_{\mathrm{FWHM}} / \sqrt{8 \ln 2}=1.235 \times$ $10^{-4} \theta_{\text {FWHM }}$, and $\theta_{\text {FWHM }}$ is the full width half maximum (FWHM) of the beam, assumed Gaussian, in arcmin. For a given mission lifetime, the noise per pixel in thermodynamic temperature is given by

$\sigma_{\text {pix }}=\frac{\Delta T}{\sqrt{n_{\text {rad }} \tau_{\text {pix }}}}$,

where $\Delta T$ is the sensitivity of each radiometer of an array with $n_{\text {rad }}$ elements, and

$\tau_{\text {pix }}=\frac{\tau_{\text {mission }}}{N_{\text {pix }}}=\frac{\tau_{\text {mission }}}{4 \pi / \theta_{\text {FWHM }}^{2}}$

is the integration time per resolution element in the sky.

\subsubsection{Angular resolution}

The basic scientific requirement for the Planck angular resolution is to provide approximately $10^{\prime}$ beams in the minimum foreground window and to achieve up to $5^{\prime}$ in the highest frequency channels. This led to a telescope in the $1.5 \mathrm{~m}$ aperture class to ensure the desired resolution with an adequate rejection of straylight contamination (Mandolesi et al. 2000a; Villa et al. 2002). In general, a trade-off occurs between main-beam resolution (half-power beam width, HPBW) and the illumination by the feeds of the edges of both the primary and sub-reflector (edge taper), which in turn drives the stray-light contamination effect. An edge taper $>30 \mathrm{~dB}$ at an angle of $22^{\circ}$ and an angular resolution of $14^{\prime}$ at $70 \mathrm{GHz}$ were set as design specifications for LFI. Detailed calculations taking into account the location of the feeds in the focal plane and the telescope optical performance (Sandri et al. 2010) showed that angular resolutions of $\sim 13^{\prime}$ are achieved for the $70 \mathrm{GHz}$ channels, while at lower frequencies we expect $24^{\prime}-28^{\prime}$ at $44 \mathrm{GHz}$ (depending on feed) and $\sim 33^{\prime}$ at $30 \mathrm{GHz}$ (see also Sect. 7).

\subsubsection{Sensitivity}

To specify the noise per frequency channel, we adopted the general criterion of uniform sensitivity per equivalent pixel. In the early design phases, based on extrapolation of previous technological progress, we set a noise specification $\Delta T_{30} / T=3 \times 10^{-6}$ (or $\Delta T_{30}=8 \mu \mathrm{K}$, thermodynamic temperature) for a reference pixel $\Delta \theta_{30} \equiv 30^{\prime}$ at all frequencies. We also considered "goal" sensitivities of $\Delta T_{30}=6 \mu \mathrm{K}$ per reference pixel, i.e., lower by $25 \%$.

With an array of $n_{\mathrm{rad}, v}$ radiometers at frequency $v$ a sky pixel will be observed, on average, for an integration time

$\tau_{\mathrm{tot}, v}=\frac{n_{\mathrm{rad}, v} \theta_{\mathrm{FWHM}, v}^{2}}{4 \pi} \tau_{\text {mission }}$

Assuming a 15 month survey, for $\Delta T_{30}=8 \mu \mathrm{K}$ the sensitivity per pixel for a 1 -s integration time is given by $\delta T_{1 \mathrm{~s}} \simeq 120 \mu \mathrm{K} \times$ $\sqrt{n_{\mathrm{rad}, v}}$. We chose $n_{\mathrm{rad}, v}$ to compensate for the higher noise temperatures at higher frequencies, while ensuring an acceptable heat load in the Planck focal plane unit (see Sect. 2.3.2) and allocate 4 radiometers at $30 \mathrm{GHz}, 6$ at $44 \mathrm{GHz}$, and 12 at $70 \mathrm{GHz}$.

As we describe in detail in Sect. 3, the LFI receivers are coupled in pairs to each feed horn $\left(n_{\text {rad }}=2 n_{\text {feeds }}\right)$ through an orthomode transducer. Thus the LFI design is such that all channels
Table 1. LFI specifications for sensitivity ${ }^{a}$ and angular resolution.

\begin{tabular}{lccc}
\hline \hline & $30 \mathrm{GHz}$ & $44 \mathrm{GHz}$ & $70 \mathrm{GHz}$ \\
\hline$\Delta T_{30}[\mu \mathrm{K}]^{b} \ldots \ldots \ldots \ldots$ & 8 & 8 & 8 \\
$\Delta T_{30} / T \ldots \ldots \ldots \ldots \ldots$ & $3 \times 10^{-6}$ & $3 \times 10^{-6}$ & $3 \times 10^{-6}$ \\
Angular resolution $\left[^{\prime}\right] \ldots$ & 33 & 24 & 14 \\
$\Delta T / T$ per pixel $\ldots \ldots \ldots$ & $2.6 \times 10^{-6}$ & $3.6 \times 10^{-6}$ & $6.2 \times 10^{-6}$ \\
$N_{\text {feeds }} \ldots \ldots \ldots \ldots \ldots \ldots$ & 2 & 3 & 6 \\
$N_{\text {radiometers }} \ldots \ldots \ldots \ldots$ & 4 & 6 & 12 \\
$\delta T_{1 \mathrm{~s}}^{\mathrm{A}}\left[\mu \mathrm{K} \mathrm{s} \mathrm{s}^{1 / 2}\right]^{c} \ldots \ldots \ldots \ldots$ & 234 & 278 & 365 \\
$\Delta v_{\text {eff }}[\mathrm{GHz}] \ldots \ldots \ldots \ldots$ & 6 & 8.8 & 14 \\
$T_{\text {sys }}[\mathrm{K}]^{d} \ldots \ldots \ldots \ldots \ldots$ & 10.7 & 16.6 & 29.2 \\
\hline
\end{tabular}

Notes. ${ }^{(a)}$ Sensitivities per pixel are specified for a nominal mission survey time of 15 months; ${ }^{(b)} \Delta T_{30}$ indicates the noise per $30^{\prime}$ reference pixel; ${ }^{(c)}$ antenna temperature; ${ }^{(d)}$ thermodynamic temperature.

are inherently sensitive to polarisation. The sensitivity to $Q$ and $U$ Stokes parameters is lower than the sensitivity to total intensity $I$ by a factor $\sqrt{2}$ since the number of channels per polarisation is only half as great. To optimise the LFI sensitivity to polarisation, the location and orientation of the LFI radiometers in the focal plan follows well-defined constraints that are described in Sect. 4. In Table 1 we summarise the main requirements for LFI sensitivity, angular resolution, and the nominal LFI design characteristics.

\subsection{Sensitivity budget}

For an array of coherent receivers, each with typical bandwidth $\Delta v$ and noise temperature $T_{\text {sys }}$, observing a sky antenna temperature $T_{\mathrm{A}, \mathrm{Sky}}$, the average white noise per pixel (in antenna temperature) will be

$\delta T_{\text {pix }, \mathrm{A}}=k_{R} \frac{T_{\text {sys }}+T_{A, \text { Sky }}}{\sqrt{\Delta v_{\text {eff }} \cdot \tau_{\text {tot }}}}$,

where $k_{R}=\sqrt{2}$ for the LFI pseudo-correlation receivers. Therefore, for a required $\delta T_{\text {pix,A }}$, the 1-s sensitivity (in antenna temperature) of each radiometer must be

$\delta T_{1 \mathrm{~s}, \mathrm{~A}}(\mu \mathrm{K} \sqrt{s})<\delta T_{\mathrm{pix}, \mathrm{A}} \sqrt{n_{\mathrm{rad}} \frac{\tau_{\text {mission }}}{N_{\mathrm{pix}}}}$,

where $N_{\text {pix }}=4 \pi / \theta_{\mathrm{FWHM}}^{2}$ is the number of pixels.

\subsubsection{Bandwidth and system noise}

A first breakdown for contributions to LFI sensitivity is between system temperature and effective bandwidth. Each radiometer is characterised by a spectral response $g(v)$ that is determined by the overall spectral response of the system including amplifiers, waveguide components, and filters. We define the radiometer effective bandwidth as

$\Delta v_{\mathrm{eff}}=\frac{\left[\int_{0}^{\infty} g(v) \mathrm{d} v\right]^{2}}{\int_{0}^{\infty} g^{2}(v) \mathrm{d} v}$

In general, therefore, ripples in the band tend to narrow the ideal rectangular equivalent bandwidth. In practice, the effective bandwidth is limited by waveguide components, filters and in-band gain ripples. Extrapolating available technology further, we assume for LFI a goal effective bandwidth of $20 \%$ of the central frequency. Equation (5) then leads to requirements on $T_{\text {sys }}$ of $\sim 10 \mathrm{~K}$ at $30 \mathrm{GHz}$, and $\sim 30 \mathrm{~K}$ at $70 \mathrm{GHz}$ (see Table 1). 
Table 2. Sensitivity budget for LFI units.

\begin{tabular}{|c|c|c|c|c|}
\hline Symbol & Name & $30 \mathrm{GHz}$ & $44 \mathrm{GHz}$ & $70 \mathrm{GHz}$ \\
\hline$L_{\text {Feed }} L_{\text {OMT }}[\mathrm{dB}]$ & Feed + OMT insertion loss $\ldots \ldots \ldots$ & -0.25 & -0.25 & -0.25 \\
\hline$T_{\mathrm{FE}}[\mathrm{K}]$ & FEM noise temperature ......... & 8.6 & 14.1 & 25.7 \\
\hline$T_{\mathrm{Feed}}+T_{\mathrm{OMT}}+T_{\mathrm{FE}}[\mathrm{K}]$ & & 10.4 & 16.2 & 28.5 \\
\hline$G_{\mathrm{FE}}[\mathrm{dB}]$ & FEM gain & $>30$ & $>30$ & $>30$ \\
\hline$L_{\mathrm{WG}}[\mathrm{dB}]$ & Waveguide insertion loss . & -2.5 & -3 & -5 \\
\hline$T_{\mathrm{BE}}[\mathrm{K}]$ & BEM noise temperature . . & 350 & 350 & 450 \\
\hline$T_{\mathrm{WB}}+T_{\mathrm{BE}}[\mathrm{K}]$ & & 0.3 & 0.4 & 0.7 \\
\hline$T_{\text {sys }}[\mathrm{K}]$ & System temperature & 10.7 & 16.6 & 29.2 \\
\hline
\end{tabular}

\subsubsection{Active cooling}

These very ambitious noise temperatures can only be achieved with cryogenically cooled low-noise amplifiers. Typically, the noise temperature of current state-of-the-art cryogenic transistor amplifiers exhibit a factor of 4-5 reduction going from $300 \mathrm{~K}$ to $100 \mathrm{~K}$ operating temperature, and another factor $2-2.5$ from $100 \mathrm{~K}$ to $20 \mathrm{~K}$. We implement active cooling to $20 \mathrm{~K}$ of the LFI front-end (including feeds, OMTs and first-stage amplification) to gain in sensitivity and to optimise the LFI-HFI thermo-mechanical coupling in the focal plane.

Because the cooling power of the $20 \mathrm{~K}$ cooler (see Sect. 5) is not compatible with the full radiometers operating at cryogenic temperature, each radiometer has been split into a $20 \mathrm{~K}$ frontend module and a $300 \mathrm{~K}$ back-end module, each carrying about half of the needed amplification ( $70 \mathrm{~dB}$ overall). This solution also avoids the serious technical difficulty of introducing a detector operating in cryogenic conditions. A set of waveguides connect the front and back-end modules; these were designed to provide sufficient thermal decoupling between the cold and warm sections of the instrument. Furthermore, low power dissipation components are required in the front-end. This is ensured by the new generation of cryogenic indium phosphide (InP) high electron mobility transistor (HEMT) devices, which yield worldrecord low-noise performance with very low power dissipation.

\subsubsection{Breakdown allocations}

While the system noise temperature, $T_{\text {sys }}$, is dominated by the performance of front-end amplifiers, additional contributions come from front-end losses and from back-end noise, which need to be minimised. For each LFI radiometer we can express the system temperature as

$T_{\mathrm{sys}}=T_{\mathrm{Feed}+\mathrm{OMT}}+T_{\mathrm{FE}}+T_{\mathrm{WGs}}+T_{\mathrm{BE}}$,

where the terms on the righthand side represent the contributions from the feed-horn/OMT, front-end module, waveguides, and back-end module. These terms can be expressed as

$$
\begin{aligned}
& T_{\mathrm{Feed}+\mathrm{OMT}}=\left(L_{\mathrm{Feed}} L_{\mathrm{OMT}}-1\right) T_{0} \\
& T_{\mathrm{FE}}=L_{\mathrm{Feed}} L_{\mathrm{OMT}} T_{\mathrm{FE}}^{\text {noise }} \\
& T_{\mathrm{WGs}}=\frac{L_{\mathrm{Feed}} L_{\mathrm{OMT}}\left(L_{\mathrm{WGs}}-1\right) T_{\mathrm{eff}}}{G_{\mathrm{FE}}} \\
& T_{\mathrm{BE}}=\frac{L_{\mathrm{Feed}} L_{\mathrm{OMT}} L_{\mathrm{WGs}} T_{\mathrm{BE}}^{\mathrm{noise}}}{G_{\mathrm{FE}}},
\end{aligned}
$$

where $T_{0}$ is the physical temperature of the front end; $T_{\text {eff }} \sim$ $200 \mathrm{~K}$ is an effective temperature of the waveguide whose exact value depends on the thermal design of the payload and radiometer interfaces; $T_{\mathrm{FE}}^{\mathrm{noise}}$ and $G_{\mathrm{FE}}$ are the noise temperature and gain of the front-end module; $T_{\mathrm{BE}}^{\mathrm{noise}}$ is the back-end module noise temperature; $L_{\mathrm{Feed}}, L_{\mathrm{OMT}}$ and $L_{\mathrm{WGs}}$ are the ohmic losses from the feed, OMT and waveguides, respectively, defined as $L_{\mathrm{X}}=10^{-L_{\mathrm{X}, \mathrm{dB}}} / 10$ (for low-loss components $L_{\mathrm{X}} \gtrsim 1$ and $\left.L_{\mathrm{X}, \mathrm{dB}} \lesssim 0\right)$.

In Table 2, we summarise the main LFI design allocations to the various elements contributing to the system temperature; these were established by taking state-of-the-art technology into account. The contribution from front-end losses, $T_{\text {Feed+OMT, }}$ is reduced to $\sim 15 \%$ by cooling the feeds and OMTs to $20 \mathrm{~K}$ and by using state-of-the-art low-loss waveguide components. Also, by requiring $30 \mathrm{~dB}$ of gain in the radiometer front-end, noise temperatures for the back-end module of $\$ 500 \mathrm{~K}$ (leading to $T_{\mathrm{BE}} \lesssim 0.5 \mathrm{~K}$ ) can be acceptable, which allows the use of standard GaAs HEMT technology for the ambient temperature amplification. More detailed design specifications for each component are given in Sect. 4 as we describe the instrument in more detail.

\subsection{Stability}

Considering perturbations to ideal radiometer stability, the minimum detectable temperature variation of a coherent receiver is given by

$\delta T(f)=k_{R} T_{\text {sys }} \sqrt{\frac{1}{\tau \cdot \Delta v_{\mathrm{eff}}}+\left[\frac{\delta G_{T}(f)}{G_{T}}\right]^{2}}$,

where $\delta G_{T}(f) / G_{T}$ represents the contribution from amplifier gain and noise temperature fluctuations at post-detection sampling frequency $f$. HEMT amplifiers are known to exhibit significant $1 / f$ noise, caused by the presence of traps in the semiconductor (Jarosik 1996), which would spoil the measurement if not suppressed. Amplifier fluctuations show a characteristic power spectral density $P(f) \propto 1 / f^{\alpha}$ with $\alpha \approx 1$, so that the noise power spectral density is given by

$P(f) \approx \sigma^{2}\left[1+\left(\frac{f_{\mathrm{k}}}{f}\right)^{\alpha}\right]$,

where $\sigma^{2}$ represents the white noise limit and the kneefrequency, $f_{\mathrm{k}}$, is the frequency at which the white noise and $1 / f$ components give equal contributions to the power spectrum (see Meinhold et al. 2009, for a detailed discussion of the LFI noise properties).

The $1 / f$ noise component not only degrades the sensitivity but also introduces spurious correlations in the time-ordered data and sky maps. The reference frequency used to set a requirement on the knee frequency for LFI is the spacecraft spin frequency, $1 \mathrm{rpm}$, or $17 \mathrm{mHz}$. However, detailed analyses (Maino et al. 2002; Keihänen et al. 2004) have shown that, for the Planck 
scanning strategy, a higher knee frequency $\left(f_{\mathrm{k}}<50 \mathrm{mHz}\right)$ is acceptable as robust destriping and map making algorithms can be successfully applied to suppress the effects of low-frequency fluctuations. Because a total power HEMT receiver would have typical knee frequencies of 10 to $100 \mathrm{~Hz}$, a very efficient differential design is needed for LFI to meet the $50 \mathrm{mHz}$ requirement.

\subsection{Systematic effects}

Throughout the design and development of LFI a key driver has been the minimisation and control of systematic effects, i.e., deviations from the signal that would be produced by an instrument with axially symmetric Gaussian beams, with ideal pointing and pure Gaussian white noise. These include optical effects (e.g., straylight, misalignment, beam distortions), instrument intrinsic effects (e.g., non-stationary and correlated noise features such as $1 / f$ noise, spikes, glitches, etc.), thermal effects (e.g., temperature fluctuations in the front-end or other instrument interfaces), and pointing errors. In particular, the LFI receiver (discussed in Sect. 3) was designed with the primary objective of minimising the impact of $1 / f$ noise, thermal fluctuations, and systematic effects due to non-ideal receiver components.

The quantitative evaluation of various potential systematic effects required a complex iterative process involving design choices, knowledge and stability of the interfaces (with HFI and with the satellite), testing and modelling of the instrument behaviour, and simulations and simplified data analysis to evaluate the impact of each effect on the scientific output of the mission (Mennella et al. 2004). Furthermore, dedicated analyses were required to evaluate the impact of instrument non-idealities on polarisation measurements (Leahy et al. 2010).

Limits on systematic effects impacting the effective angular resolution (beam ellipticity, alignment, pointing errors) were used, together with those coming from HFI, as input to the design of the Planck telescope and focal plane, as well as to set pointing requirements at the system level. Regarding signal perturbations, for LFI we set an upper limit to the global impact of systematic effects of $<3 \mu \mathrm{K}$ per pixel at the end of the mission and after data processing. Starting from this cumulative limit, we defined a breakdown of contributions from various kinds of effects (Table 3), and then we worked out more detailed allocations for each contribution. This provided a useful guideline for the design, development, and testing of the various LFI subsystems. For each type of systematic error we specify limits for three cases: a high-frequency component, spinsynchronous fluctuations, and periodic (non-spin-synchronous) fluctuations. High-frequency contributions $(\gg 0.016 \mathrm{~Hz})$ can be considered as random fluctuations and added in quadrature to the radiometers' white noise. As a goal, the overall noise increase due to random effects other than radiometer white noise should be less than $10 \%$. Spin synchronous $(0.016 \mathrm{~Hz})$ components are not damped by scanning redundancy, and impose the most stringent limits on systematic effects. Periodic fluctuations on time scales other than the satellite spin are damped with an efficiency that depends on the characteristic time scale of the effect (see Mennella et al. 2002a, for quantitative analysis). For $1 / f$ and thermal non-spin-synchronous fluctuations, affecting long time scales, we set the acceptable limits on systematic effects assuming that a consolidated destriping algorithm is applied to the data (Maino et al. 1999, 2002).

High level allocations for signal perturbation effects are indicated in Table 3. The meaning of some of these contributions will become clearer as we provide a description of the design
Table 3. Top-level systematic error budget (peak-to-peak values).

\begin{tabular}{|c|c|c|c|}
\hline Source & $\begin{array}{l}\text { Random }^{a} \\
\delta T \text { fraction }\end{array}$ & $\begin{array}{c}\text { Spin synch } \\
{[\mu \mathrm{K}]}\end{array}$ & $\begin{array}{c}\text { Periodic }^{a} \\
{[\mu \mathrm{K}]}\end{array}$ \\
\hline External straylight ... & & 1 & \\
\hline Internal straylight .. & 0.045 & 1 & 0.9 \\
\hline $4 \mathrm{~K}$ load $\ldots . . . \ldots \ldots$ & 0.025 & 1 & $0.6(11)$ \\
\hline Thermal fluctuations & 0.03 & 0.8 & $1.1(11)$ \\
\hline Front end $1 / f \ldots \ldots$ & $0.25(0.34)$ & .. & $\ldots$ \\
\hline Back end $1 / f \ldots \ldots$ & $0.35(0.48)$ & $\ldots$ & $\ldots$ \\
\hline DC electronics ..... & 0.04 & $\cdots$ & $\cdots$ \\
\hline Quantisation ....... & 0.01 & $\cdots$ & $\cdots$ \\
\hline Total $\ldots \ldots \ldots \ldots \ldots$ & 0.44 & 1.9 & 1.5 \\
\hline Noise increase $\ldots .$. & 1.091 & & \\
\hline
\end{tabular}

Notes. ${ }^{(a)}$ For $1 / f$ and thermal periodic fluctuations the allocated limits are residuals after consolidated software removal techniques are applied to the data (numbers in parenthesis give the effect before removal).

solutions adopted for the LFI instrument and its interfaces (Sects. 4 to 7 ).

\section{Instrument concept}

The heart of the LFI instrument is an array of 22 differential receivers based on cryogenic high-electron-mobility transistor (HEMT) amplifiers. Cooling of the front end is achieved by a closed-cycle hydrogen sorption cooler (Morgante et al. 2009), with a cooling power of about $1 \mathrm{~W}$ at $20 \mathrm{~K}$, which also provides $18 \mathrm{~K}$ pre-cooling to the HFI.

Radiation from the sky intercepted by the Planck telescope is coupled to 11 corrugated feed horns, each connected to a double-radiometer system, the so-called radiometer chain assembly (RCA, see Fig. 1). The complete LFI array, including 11 RCAs and 22 radiometers, is called the radiometer array assembly (RAA).

\subsection{Radiometer chain assemblies}

Downstream of each feedhorn, an orthomode transducer (OMT) separates the signal into two orthogonal polarisations with minimal losses and cross talk. Two parallel, independent radiometers are connected to the output ports of the OMT, thus preserving the polarisation information. Each radiometer pair is split into a front end module (FEM) and a back-end module (BEM) to minimise power dissipation in the actively cooled front end. A set of composite waveguides connect the FEM and the BEM.

The stringent stability requirements are obtained with a pseudo-correlation receiver in which the signal from the sky is continuously compared with the signal from a blackbody reference load. The loads, one for each radiometer, are cooled to approximately $4.5 \mathrm{~K}$ by a Stirling cooler that provides the second pre-cooling stage for the HFI bolometers. As we show in detail in Sect. 3.3, each radiometer has two internal symmetric legs, so that each RCA comprises four waveguides connecting the FEM and the BEM and four detector diodes.

Each RCA is designated by a consecutive number (see Sect. 4.1.1). In each RCA, the radiometer connected to the main arm of the OMT is called R0, and the one connected to the side arm is called R1, as shown in Fig. 1. The two detectors in radiometer R0 are named (M-00, M-01), while those in radiometer $\mathrm{R} 1$ are named $(\mathrm{S}-10, \mathrm{~S}-11)$. 

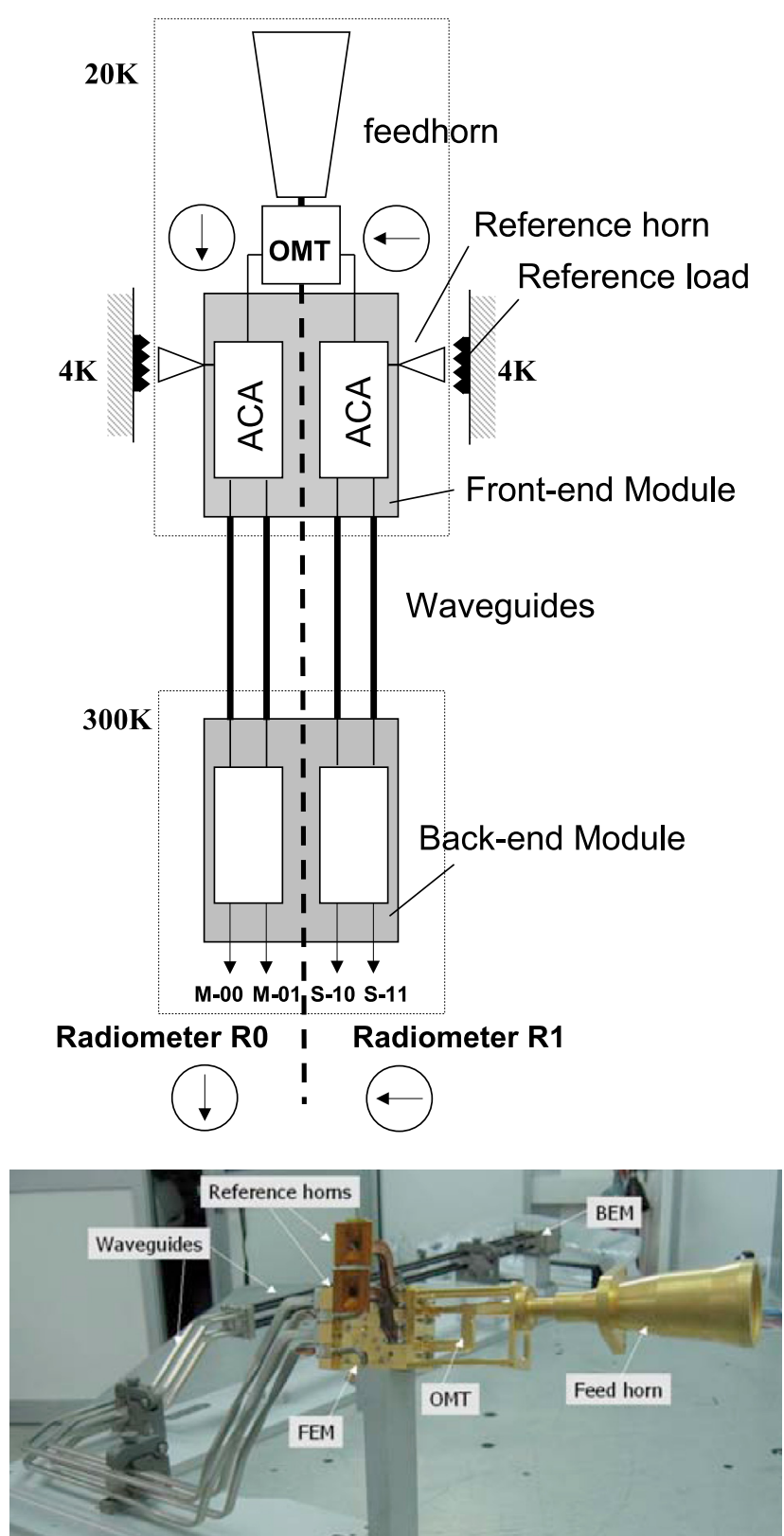

Fig. 1. Top: schematic of a radiometer chain assembly (RCA). The LFI array has 11 RCAs, each comprising two radiometers carrying the two orthogonal polarisations. The RCA is constituted by a feed horn, an orthomode transducer (OMT), a front-end module (FEM) operated at $20 \mathrm{~K}$, a set of four waveguides that connect FEM to the back-end module (BEM). The notations "0" and "1" for the two radiometers in the RCA denote the branches downstream of the main and side arms of the OMT, respectively. Each amplifier chain assembly (ACA) comprises a cascaded amplifier and a phase switch. Bottom: picture of a $30 \mathrm{GHz}$ RCA integrated before radiometer-level tests.

\subsection{Radiometer array assembly}

A schematic of the radiometer array assembly (RAA), is shown in Fig. 2. Each RCA has been integrated and tested separately, and then mounted on the RAA without de-integration to ensure stability of the radiometer characteristics after calibration at RCA level (Villa et al. 2010).

A "mainframe" supports the LFI $20 \mathrm{~K}$ front end (with feeds, OMTs, and FEMs) and interfaces the HFI $4 \mathrm{~K}$ front-end box in the central portion of the focal plane. The HFI $4 \mathrm{~K}$ box is linked

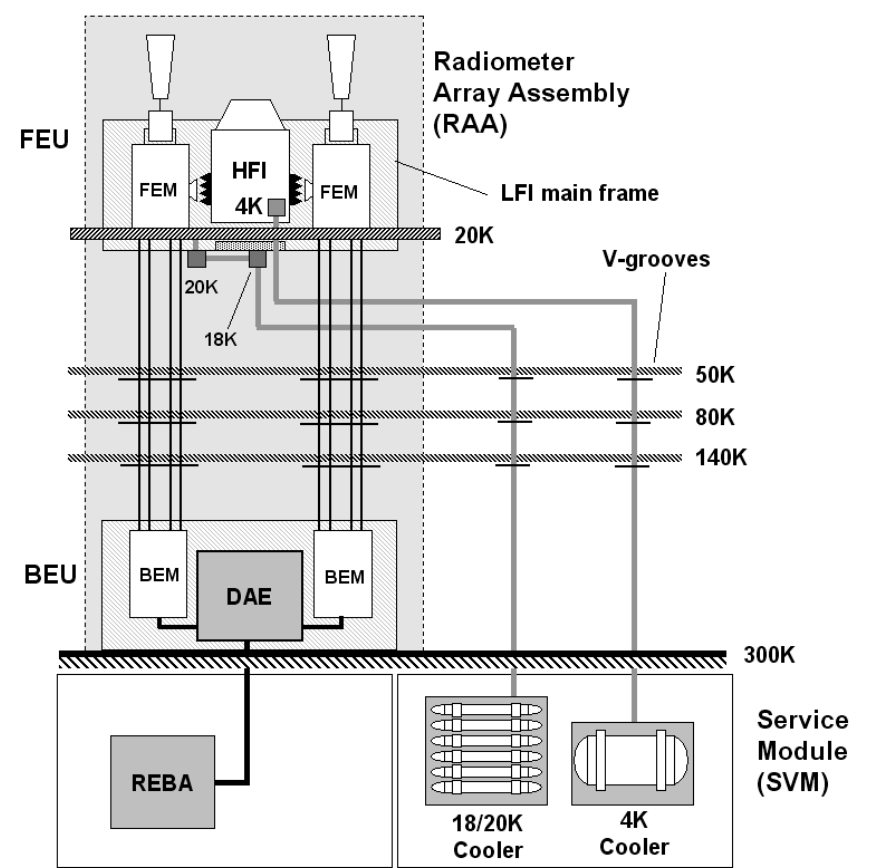

Fig. 2. Schematic of the LFI system displaying the main thermal interfaces with the V-grooves and connections with the $20 \mathrm{~K}$ and $4 \mathrm{~K}$ coolers. Two RCAs only are shown in this scheme. The radiometer array assembly (RAA) is represented by the shaded area and comprises the front-end unit (FEU) and back-end unit (BEU). The entire LFI RAA includes 11 RCAs, with 11 feeds, 22 radiometers, and 44 detectors.

to the $20 \mathrm{~K}$ LFI mainframe with insulating struts and provides the thermal and mechanical interface to the LFI reference loads. Forty-four waveguides connect the LFI front-end unit (FEU) to the back-end unit (BEU), which is mounted on the top panel of the Planck service module (SVM) and is maintained at a temperature of $\sim 300 \mathrm{~K}$. The BEU comprises the eleven BEMs and the data acquisition electronics (DAE) unit. After on-board processing, provided by the radiometer box electronics assembly (REBA), the compressed signal is down-linked to the ground station with housekeeping data.

A major design driver has been to ensure acceptably low conductive and radiative parasitic thermal loads at the $20 \mathrm{~K}$ stage, particularly those introduced by the waveguides and cryoharness. As we discuss below, sophisticated design solutions were implemented for these units. In addition, three thermal sinks were used to largely reduce the parasitic loads in the $20 \mathrm{~K}$ stage, and these are the three conical shields (V-grooves) introduced in the Planck payload module to thermally isolate the cold telescope enclosure from the SVM at $\sim 300 \mathrm{~K}$ (Tauber et al. 2010a). The V-grooves also provide multiple precool temperatures to all of the Planck coolers, as well as intercepting parasitics from the cooler piping and HFI equipment. The three $\mathrm{V}$-grooves are expected to reach in-flight temperatures of approximately $170 \mathrm{~K}, 100 \mathrm{~K}$, and $50 \mathrm{~K}$.

The FEU is aligned in the focal plane of the telescope and supported by a set of three thermally insulating bipods attached to the telescope structure. The back-end unit is fixed on top of the Planck service module, below the lower V-groove. In Fig. 3 we show a detailed drawing of the RAA, including an exploded view showing its main subassemblies and units. After integration, the RAA was first tested in a dedicated cryo-facility (Mennella et al. 2010) for instrument level tests (Fig. 4), and then inserted into 


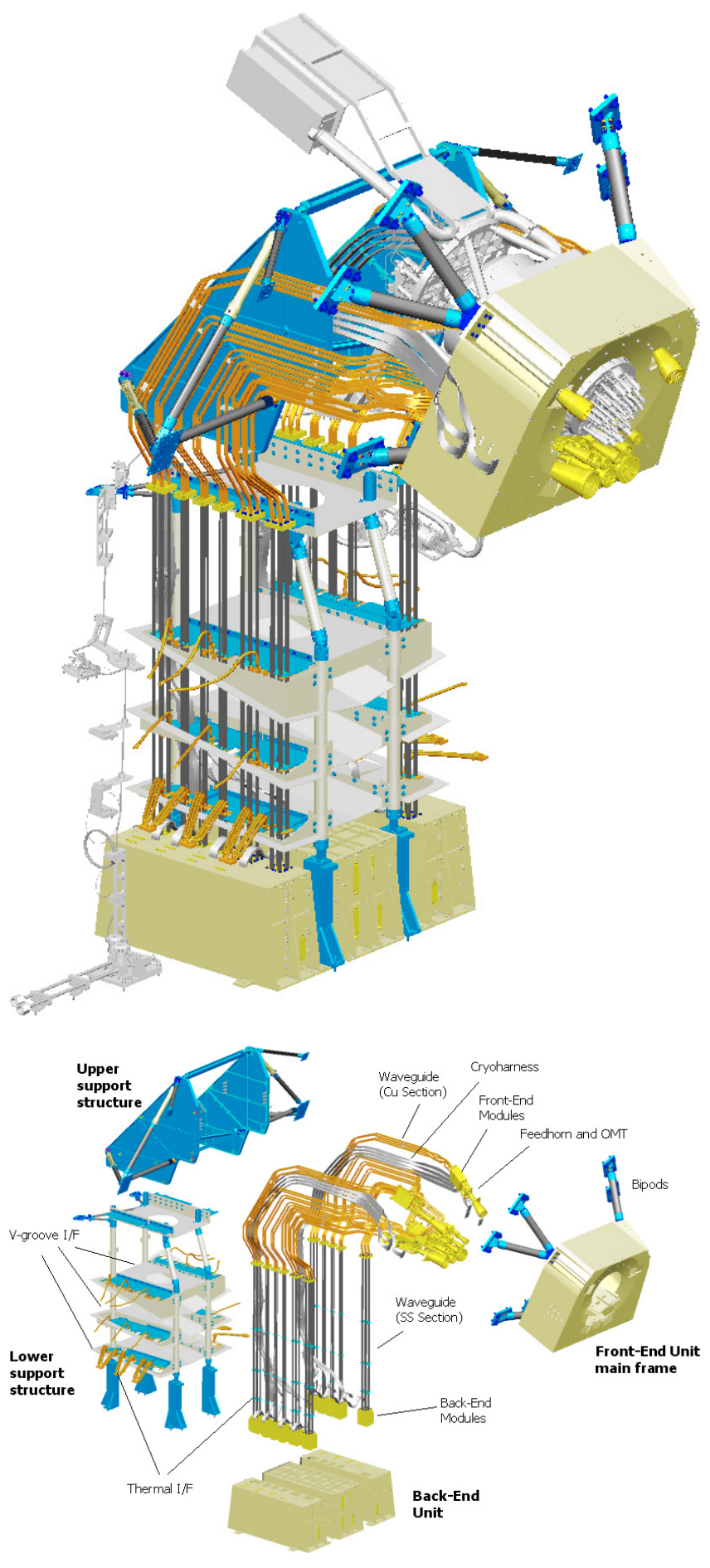

Fig. 3. LFI RAA. Top: drawing of the integrated instrument showing the focal plane unit, waveguide bundle and back-end unit. The elements that are not part of LFI hardware (HFI front-end, cooler pipes, thermal shields) are shown in light grey. Bottom: more details are visible in the exploded view, as indicated in the labels.

the payload module after integrating the HFI $4 \mathrm{~K}$ box. Figure 5 shows the LFI within the Planck satellite.

\subsection{Receiver design}

The LFI receivers are based on an additive correlation concept, or pseudo-correlation, analogous to schemes used in previous applications in early works (Blum 1959), as well as in

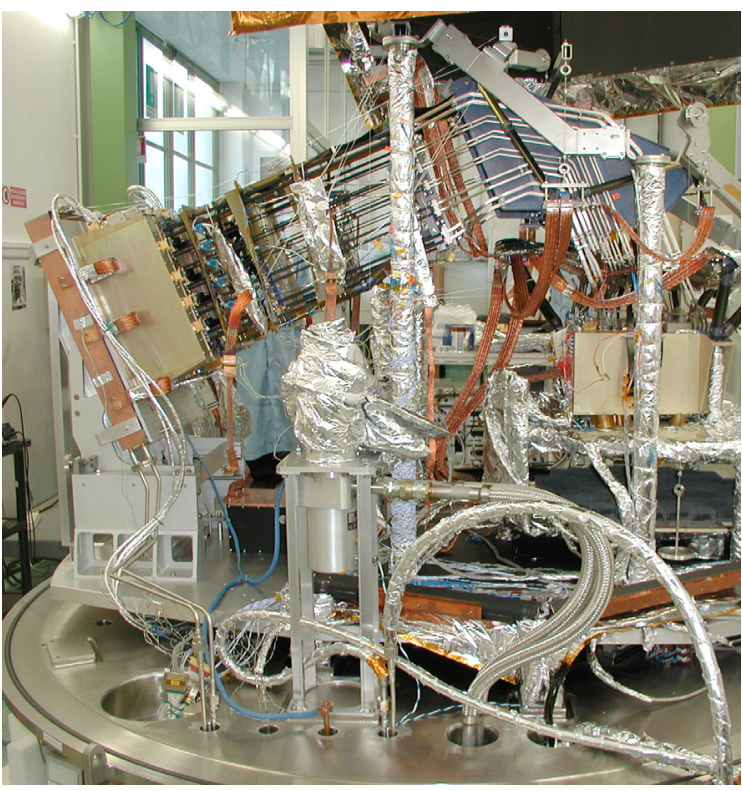

Fig. 4. The LFI instrument in the configuration for instrument level test cryogenic campaign.

recent CMB experiments (Staggs et al. 1996; Jarosik et al. 2003). The LFI design introduces new features that optimise stability and immunity to systematics within the constraints imposed by cryogenic operation and by integration into a complex payload such as Planck. The FEM contains the most sensitive part of the receiver, where the pseudo-correlation scheme is implemented, while the BEM provides further RF amplification and detection.

In each radiometer (Fig. 6), after the OMT, the voltages of the signal from the sky horn, $x(t)$, and from the reference load, $y(t)$, are coupled to a $180^{\circ}$ hybrid that yields the mixed signals $(x+y) / \sqrt{2}$ and $(x-y) / \sqrt{2}$ at its two output ports. These signals are then amplified by the cryogenic low-noise amplifiers (LNAs) characterised by noise voltage, gain, and phase $n_{F_{1}}, g_{F_{1}}$, $\phi_{F_{1}}$ and $n_{F_{2}}, g_{F_{2}}, \phi_{F_{2}}$. One of the two signals then runs through a switch that shifts the phase between 0 and $180^{\circ}$ at a frequency of $4096 \mathrm{~Hz}$. A second phase switch is mounted for symmetry and redundancy on the other radiometer leg, but it does not introduce any switching phase shift. The signals are then recombined by a second $180^{\circ}$ hybrid coupler, thus producing an output, which is a sequence of signals proportional to $x(t)$ and $y(t)$ alternating at twice the phase switch frequency.

In the back-end modules (Fig. 6), the RF signals are further amplified in the two legs of the radiometers by room temperature amplifiers characterised by noise voltage, gain and phase $n_{B_{1}}$, $g_{B_{1}}, \phi_{B_{1}}$ and $n_{B_{2}}, g_{B_{2}}, \phi_{B_{2}}$. The signals are filtered and then detected by square-law detector diodes. A DC amplifier then boosts the signal output, which is connected to the data acquisition electronics. The sky and reference load DC signals are integrated, digitised, and then transmitted to the ground as two separated streams of sky and reference load data.

The sky and reference load signals recombined after the second hybrid in the FEM have highly correlated $1 / f$ fluctuations. This is because, in each radiometer leg, both the sky and the reference signals undergo the same instantaneous fluctuations due to LNAs intrinsic instability. Furthermore, the fast modulation drastically reduces the impact of $1 / f$ fluctuations coming from the back-end amplifiers and detector diodes, since the switch rate $\sim 4 \mathrm{kHz}$ is much higher than the $1 / f$ knee frequency of the BEM components. By taking the difference between the 

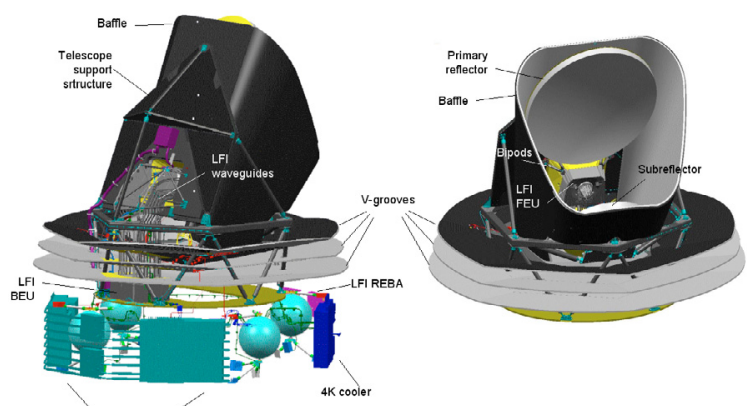

$20 \%$ sorption coolór compress ors
(nominal and re dundant)

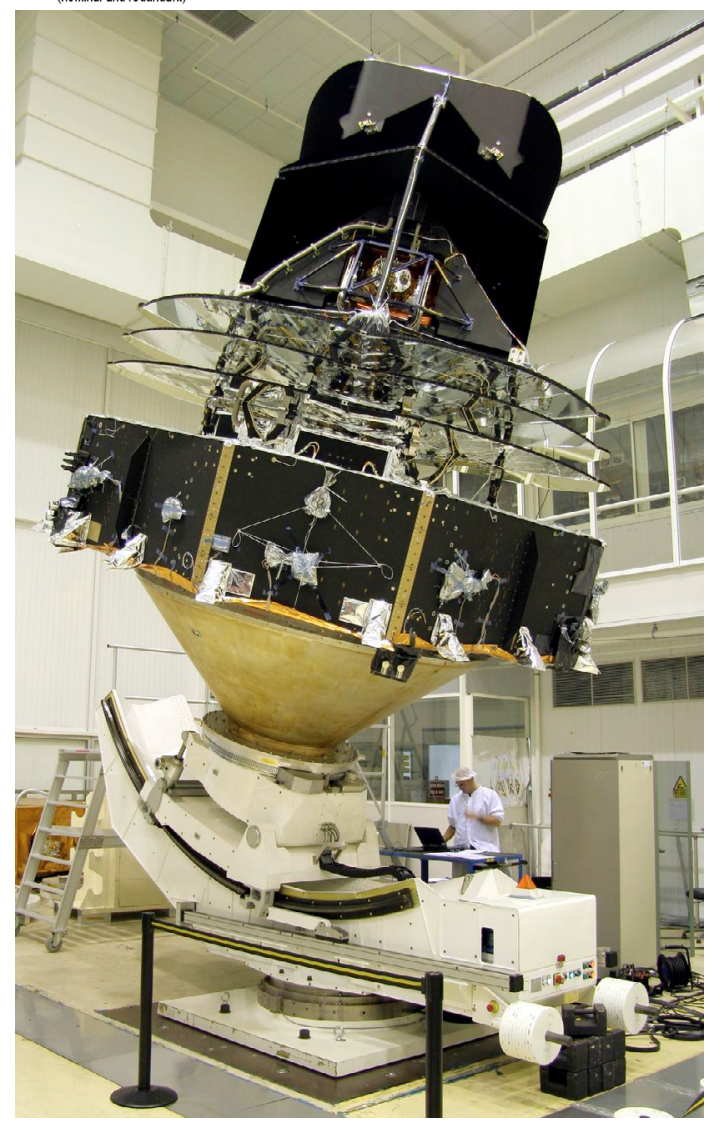

Fig. 5. Top: schematic of the Planck satellite showing the main interfaces with the LFI RAA on the spacecraft. Bottom: back view of Planck showing the RAA integrated on the PPLM. The LFI Back-end unit is the box below the lowest V-groove and resting on the top panel of the SVM.

DC output voltages $V_{\text {sky }}$ and $V_{\text {load }}$, therefore, the $1 / f$ noise is highly reduced.

Differently from the WMAP receivers, the LFI phase switches and second hybrids have been placed in the front end. This allows full modularity of the FEMs, BEMs, and waveguides, which in turns simplifies the integration and test procedure. Furthermore, this design does not require that the phase be preserved in the waveguides, a major advantage given the complex routing imposed by the LFI-HFI integration and the potentially significant thermo-elastic effects from the cryogenic interfaces in the Planck payload.

In principle, for a null differential output corresponding to a perfectly balanced system, fluctuations would be fully suppressed in the differenced data. In practice, for LFI, a residual offset will be necessarily present due to input asymmetry between the sky arm $(\sim 2.7 \mathrm{~K}$ from the sky, plus $\sim 0.4 \mathrm{~K}$ from the reflectors) and the reference load arm (with physical

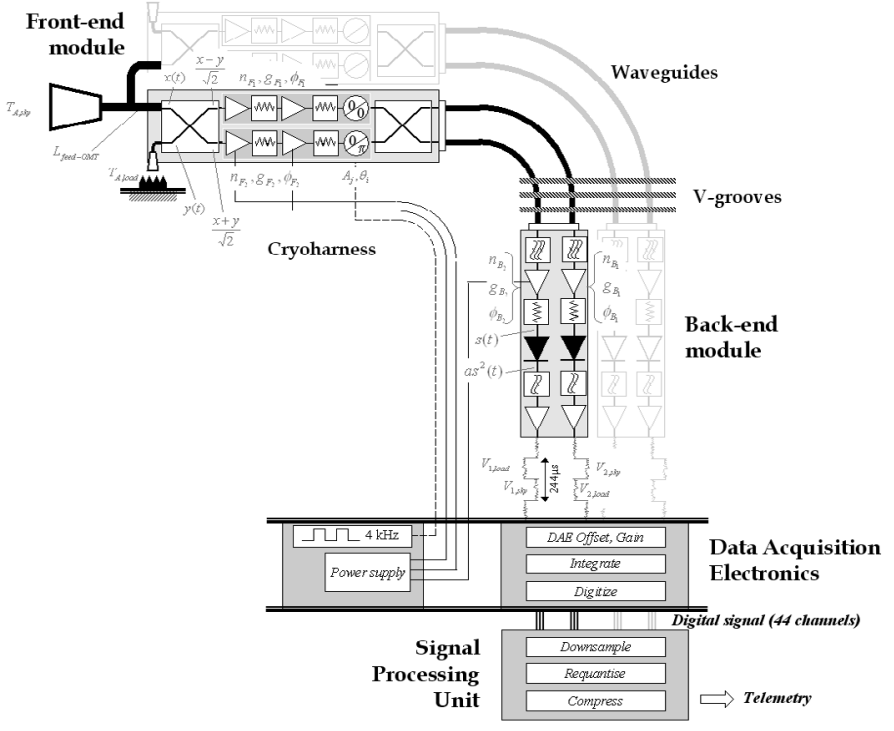

Fig. 6. LFI receiver scheme, shown in the layout of a radiometer chain assembly (RCA). Some details in the receiver components (e.g., attenuators, filters, etc.) differ slightly for the different frequency bands.

temperature $\sim 4.5 \mathrm{~K}$ ), plus a small contribution from inherent radiometer asymmetry. To compensate for this effect, a gain modulation factor $r$ is introduced in software to null the output by taking the difference $\bar{p}=V_{\text {sky }}-r V_{\text {load }} \approx 0$. In the next section, we discuss the signal model in more detail.

\subsubsection{Signal model}

If $x(t)$ and $y(t)$ are the input voltages at each component, then the transfer functions for the hybrids, the front-end amplifiers, the phase switches, and the back-end amplifiers can be written, respectively, as

$$
\begin{aligned}
& f_{\text {hybrid }}:\{x, y\} \rightarrow\left\{\frac{x+y}{\sqrt{2}}, \frac{x-y}{\sqrt{2}}\right\} \\
& f_{\mathrm{amp}}^{\mathrm{FE}}:\{x, y\} \rightarrow\left\{g_{F_{1}}\left(x+n_{F_{1}}\right) \mathrm{e}^{\mathrm{i} \phi_{F_{1}}}, g_{F_{2}}\left(y+n_{F_{2}}\right) \mathrm{e}^{\mathrm{i} \phi_{F_{2}}}\right\} \\
& f_{\mathrm{sw}}:\{x, y\} \rightarrow\left\{x, y \sqrt{A_{j}} \mathrm{e}^{i \theta_{j}}\right\}, j=1,2 \\
& f_{\mathrm{amp}}^{\mathrm{BE}}:\{x, y\} \rightarrow\left\{g_{B_{1}}\left(x+n_{B_{1}}\right) \mathrm{e}^{\mathrm{i} \phi_{B_{1}}}, g_{B_{2}}\left(y+n_{B_{2}}\right) \mathrm{e}^{\mathrm{i} \phi_{B_{2}}}\right\},
\end{aligned}
$$

where $\theta_{1}$ and $\theta_{2}$ are the phase shifts in the two switch states (nominally, $\theta_{1}=0$ and $\theta_{2}=180^{\circ}$ ), $n_{F}$ and $n_{B}$ represent the white noise of the front-end and back-end amplifiers, and $A_{1}$ and $A_{2}$ represent the fraction of the signal amplitude that is transmitted after the phase switch in the two states (for a lossless switch $A_{1}=A_{2}=1$ ). Based on these transfer functions and on the topology of the LFI receiver discussed above, we developed a detailed analytical description of the receiver and evaluated its susceptibility to systematic effects by studying the impact of deviation from ideal radiometer behaviour on the differenced output (Seiffert et al. 2002; Mennella et al. 2002a). In addition to the analytical treatment, a numerical model of the RCA signals has been developed (Battaglia et al. 2009).

For small phase mismatches and assuming negligible phase switch imbalance, the power output of the differenced signal after applying the gain modulation factor is given by

$\Delta p=a k \beta\left[\tilde{T}_{A, \text { sky }}(G-r I)-r \tilde{T}_{A, \text { load }}\left(G-\frac{1}{r} I\right)+(1-r) T_{\text {sys }}\right]$. 
M. Bersanelli et al.: Planck pre-launch status: Design and description of the Low Frequency Instrument
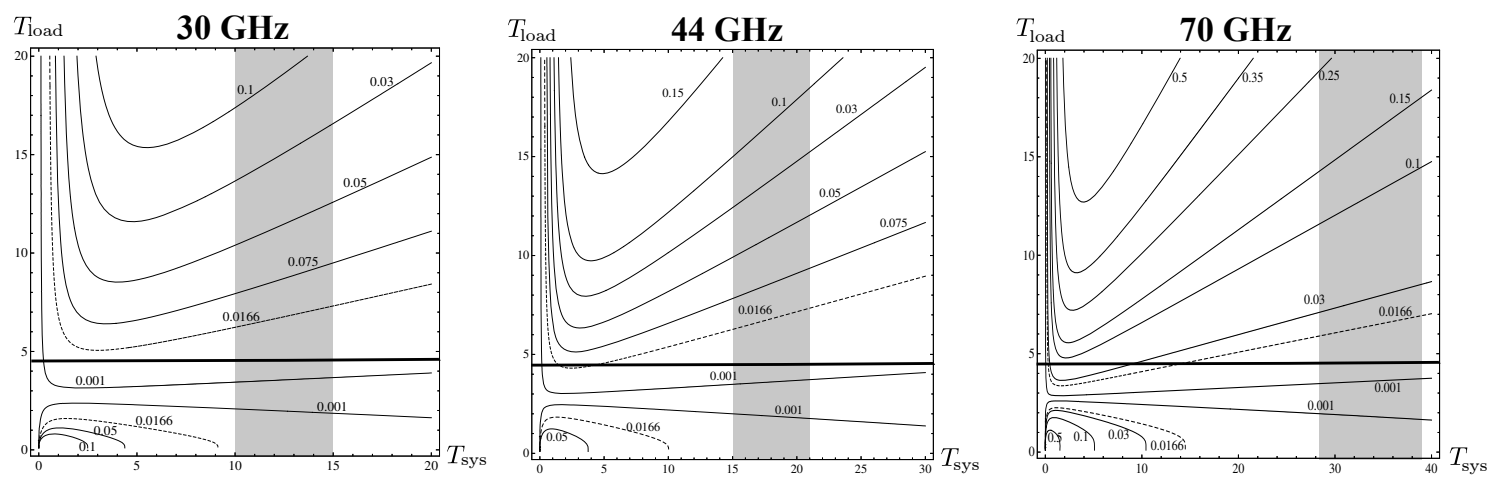

Fig. 7. Curves of equal $f_{\mathrm{k}}$ (in $\mathrm{Hz}$ ) on the plane $T_{\text {load }}\left(\mathrm{K}\right.$, thermodynamic temperature), $T_{\text {sys }}$ assuming thermodynamic sky temperature of $2.7 \mathrm{~K}$. Each panel refers to a different frequency channel. The dashed contour refers to values for which the knee frequency is equal to the spin frequency $\left(f_{\text {spin }}=0.0166 \mathrm{~Hz}\right)$. The graphs also show the range of typical LFI noise temperature values (grey area) and the nominal reference load temperature (4 K - double horizontal line).

In Eq. (12) $a$ is the proportionality constant of the square-law detector diode, and $G$ and $I$ are the effective power gain and isolation of the system:

$G \simeq \frac{1}{4} g_{B}^{2}\left(g_{F_{1}}^{2}+g_{F_{2}}^{2}+2 g_{F_{1}} g_{F_{2}}\right)$

$I \simeq \frac{1}{4} g_{B}^{2}\left(g_{F_{1}}^{2}+g_{F_{2}}^{2}-2 g_{F_{1}} g_{F_{2}}\right)$,

where $g_{B}$ is the voltage gain of the BEM in the considered channel. In Eq. (12) the temperature terms,

$\tilde{T}_{\text {sky }}=\frac{T_{\text {sky }}}{L_{\text {feed }} L_{\mathrm{OMT}}}+\left(1-\frac{1}{L_{\text {feed }} L_{\mathrm{OMT}}}\right) T_{\text {phys }}$

$\tilde{T}_{\text {load }}=\frac{T_{\text {load }}}{L_{4 \mathrm{~K}}}+\left(1-\frac{1}{L_{4 \mathrm{~K}}}\right) T_{\text {phys }}$,

represent the sky and reference load signals at the input of the first hybrid, where $L_{4 \mathrm{~K}}$ is the insertion loss of the reference horn, and $T_{\text {phys }} \simeq 20 \mathrm{~K}$ is the front-end physical temperature.

\subsubsection{Knee frequency and gain modulation factor}

For a radiometer with good isolation $(>13 \mathrm{~dB})$, as expected in a well-matched system, it follows from Eq. (12) that the power output is nullified for

$r=\frac{\tilde{T}_{\text {sky }}+T_{\text {noise }}}{\tilde{T}_{\text {load }}+T_{\text {noise }}}$.

In this case the gain fluctuations are fully suppressed and the radiometer is only sensitive to the $1 / f$ noise caused by noise temperature fluctuations, which only represents a small fraction of the amplifiers instability. For an optimal choice of the gain modulation factor, the resulting knee frequency is given by

$f_{\mathrm{k}} \simeq \Delta v\left(\frac{A(1-r) T_{\text {sys }}}{T_{\text {sky }}+T_{\text {sys }}}\right)^{2} \propto(1-r)^{2}$.

Thus, in principle, for small input offsets $\tilde{T}_{\text {sky }} \simeq \tilde{T}_{\text {load }}$ very low knee frequencies can be obtained. Figure 7 displays expected knee frequencies for parameters typical of the LFI channels as a function of noise temperature and reference load temperature, assuming ideal gain and phase match. For $T_{\text {load }} \approx 4 \mathrm{~K}$ we expect $f_{\mathrm{k}}$ to be an order of magnitude lower than for $T_{\text {load }} \approx 20 \mathrm{~K}$. This was the driver for implementing reference loads at $4 \mathrm{~K}$ at the cost of some complexity in the thermo-mechanical interfaces

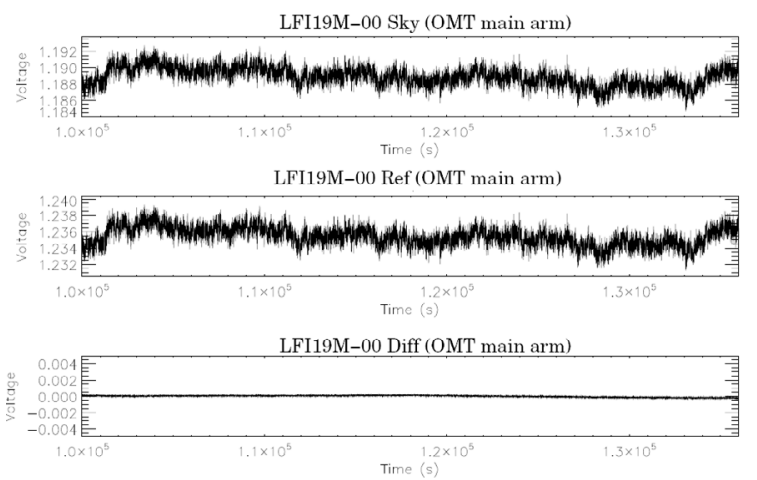

Fig. 8. Example of uncalibrated data stream from one of the 44 LFI detectors (LFI19M-00, at $70 \mathrm{GHz}$ ) recorded during instrument level tests. The upper and middle panels show the data for the sky and reference load inputs, while the lower panel shows differenced data stream with optimal gain modulation factor.

in the focal plane. It can also be shown (Mennella \& Bersanelli 2001) that the same value of $r$ that minimises the radiometer sensitivity to $1 / f$ noise is also effective in reducing the susceptibility to other systematic effects such as back-end temperature variations.

It is essential that the gain modulation factor $r$ be calculated with sufficient precision to reach the required stability. Simulations and testing show that the needed accuracy ranges from $\pm 1 \%(30 \mathrm{GHz})$ to $\pm 0.5 \%(70 \mathrm{GHz})$. This accuracy can be obtained with different methods (Mennella et al. 2003), the simplest being to evaluate the ratio of the total power output voltages averaged over a suitable time interval, $r \simeq \bar{V}_{\text {sky }} / \bar{V}_{\text {load }}$. In Fig. 8 we show, as an example, the data streams from one of the 44 LFI detectors with the two total power signals and differenced data. The LFI telemetry allocations ensure that the total power data from both the sky and reference load samples will be downloaded, so calculation of $r$ and differencing is performed on the ground.

Further suppression of common fluctuation modes, typically of thermal or electrical origin, is obtained by taking the noiseweighted average of the two detectors associated to each radiometer (Mennella et al. 2010) as well as in the differencing of the main and side arm radiometers signals when analysing data for polarisation (Leahy et al. 2010). 


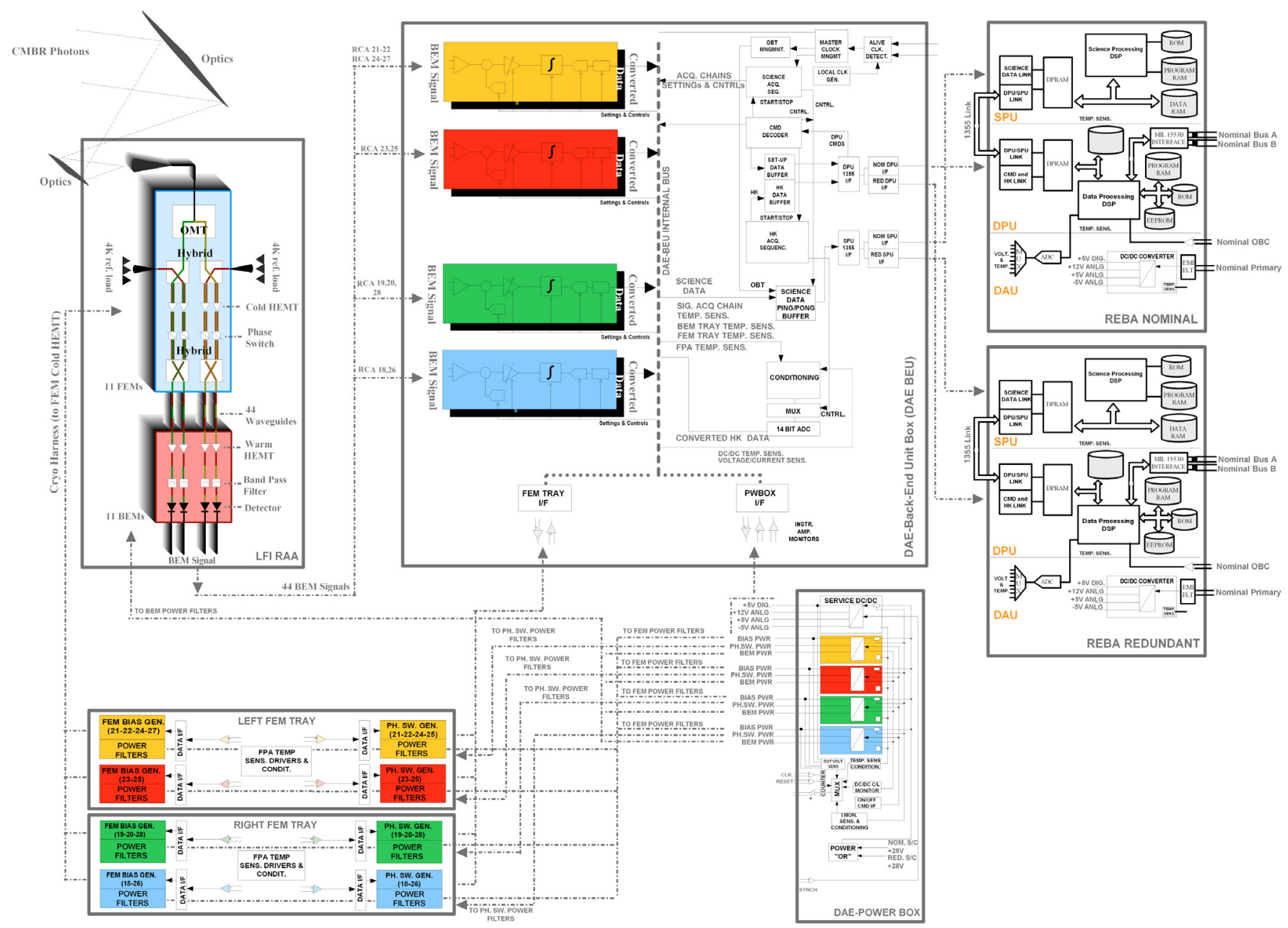

Fig. 9. Schematics of the LFI showing interconnections and details of the DAE and REBA main functions and units.

\subsubsection{Noise temperature}

The LFI radiometer sensitivity is essentially independent of the temperature of the reference loads. From Eqs. (5) and (12) it follows that, to first order, the radiometer sensitivity is

$\delta T=\sqrt{\frac{2}{\tau \cdot \Delta v}}\left(T_{\mathrm{sys}}+T_{\mathrm{sky}}\right) \sqrt{1+\eta_{L}}$,

where

$\eta_{L}=\frac{\left(T_{N, B} / G_{F}\right)^{2}}{\left(T_{\text {sys }}+T_{\text {sky }}\right)\left(T_{\text {sys }}+T_{\text {load }}\right)}$,

and $T_{N, B}$ is the noise temperature of the back-end, and $G_{F}$ the gain of the front end. For parameter values typical of LFI, we have $\eta_{L}<10^{-3}$, so that the dependence of the noise temperature on $T_{\text {load }}$ is extremely weak. The advantage of cooling the reference load to $4 \mathrm{~K}$, therefore, rests solely on better suppression of systematics, not on sensitivity.

\section{LFI configuration and subsystems}

The overall LFI system is shown schematically in Fig. 9. In this section we provide an overview of the instrument units and main subsystems. More details on the design, development and testing of the most critical components are given in companion papers that are cited below.

\subsection{The front-end unit}

\subsubsection{Focal plane design}

The disposition of the LFI feeds in the focal plane is driven by optimisation of angular resolution and by recovery of polarisation information. In addition, requirements need to be met on proper sampling of the sky and rejection of crosstalk effects.

The central portion of the Planck focal plane is occupied by the HFI front end, as higher frequency channels are more susceptible to optical aberration. The LFI feeds are located as close as possible to the focal plane centre compatible with mechanical interfaces with HFI and $4 \mathrm{~K}$ reference loads (Fig. 10). Miniaturised designs for the FEMs and OMT are implemented to allow optimal use of the focal area. The $70 \mathrm{GHz}$ feeds, most critical for cosmological science, are placed in the best location for angular resolution and low beam distortion required for this frequency (Sandri et al. 2010).

A key criterion for the feed arrangement is that the $E$ and $H$ planes as projected in the sky will allow optimal discrimination of the Stokes $Q$ and $U$ parameters. The polarisation information is obtained by differencing the signal measured by the mainarm (R0) and side-arm (R1) radiometers in each RCA, which are set to $90^{\circ}$ angle by the OMT. An optimal extraction of $Q$ and $U$ is achieved if subsets of channels are oriented in such a way that the linear polarisation directions are evenly sampled (Leahy et al. 2010). We achieve this by arranging pairs of feeds with their $E$ planes oriented at $45^{\circ}$ to each other (Fig. 11). With the 
M. Bersanelli et al.: Planck pre-launch status: Design and description of the Low Frequency Instrument
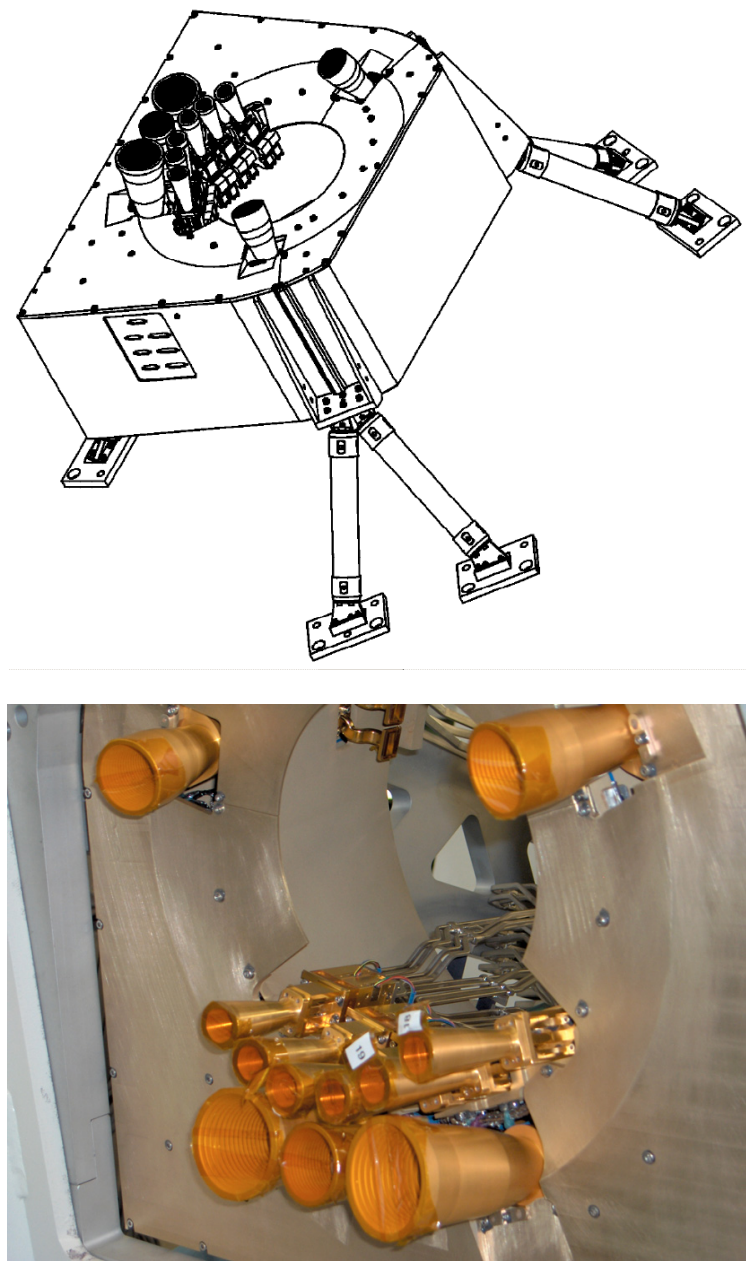

Fig. 10. Arrangement of the LFI feeds in the focal plane. Top: mechanical drawing of the main frame and focal plane elements. Shown are the bipods connecting the FPU to the telescope structure. Bottom: picture of the LFI flight model focal plane.

exception of one $44 \mathrm{GHz}$ feed, all the $E$ planes are oriented at $\pm 22.5^{\circ}$ relative to the scan direction. We also align pairs of feeds such that their projected lines of sight will follow each other in the Planck scanning strategy.

The spin axis of Planck will be shifted by $2^{\prime}$ every $\sim 45 \mathrm{~min}$ (Tauber et al. 2010a). Therefore, even for the highest LFI angular resolution, $\theta_{\mathrm{FWHM}} \simeq 13^{\prime}$ at $70 \mathrm{GHz}$, the sky is well sampled.

\subsubsection{LFI main frame}

The LFI mainframe provides thermo-mechanical support to the LFI radiometer front-end, but it also supports the HFI and interfaces to the cold end (nominal and redundant) of the $20 \mathrm{~K}$ sorption cooler. In fact, some of the key requirements on the LFI mainframe (stiffness, thermal isolation, optical alignment) are driven by the HFI rather than the LFI instrument. The mainframe is built of the aluminium alloy 6061-T6, and it is dismountable in three subunits to facilitate integration of the complete RCAs and of the HFI front-end.

The interface between the FPU and the $50 \mathrm{~K}$ payload module structure must ensure thermal isolation, as well as compliance with eigenfrequencies from launch loads. A trade-off was made to identify the proper material properties, fibre orientations, and strut inclinations. The chosen configuration was a set of three 225-mm-long CFRP T300 bipods, inclined at 50 ${ }^{\circ}$. The LFI-HFI

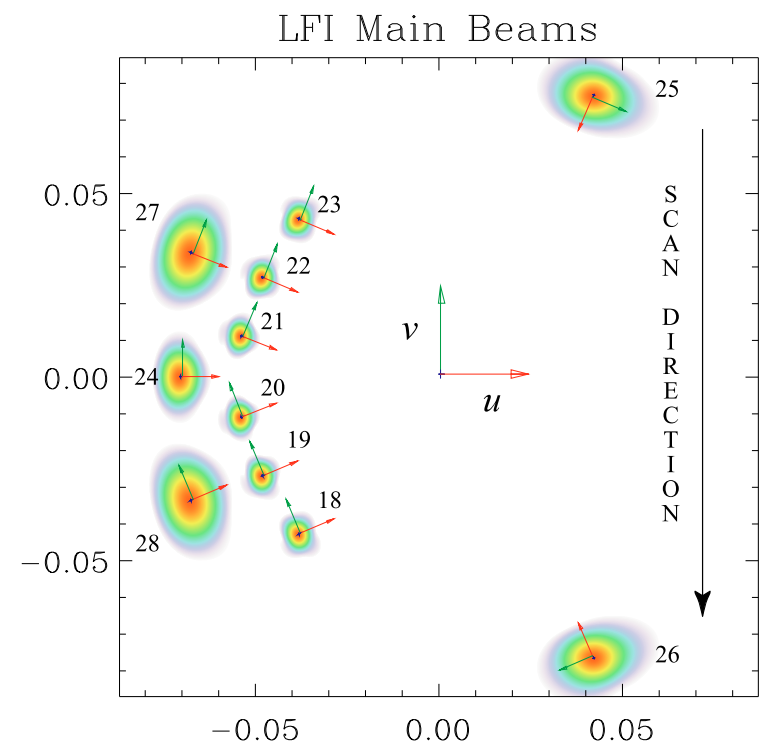

Fig. 11. Footprint of the LFI main beams on the sky and polarisation angles as seen by an observer looking towards the satellite along its optical axis. The units are $u-v$ coordinates defined as $u=\sin \theta_{\mathrm{bf}} \cos \phi_{\mathrm{bf}}$ and $v=\sin \theta_{\mathrm{bf}} \sin \phi_{\mathrm{bf}}$, where $\theta_{\mathrm{bf}}$ and $\phi_{\mathrm{bf}}$ refer to each main beam frame (Sandri et al. 2010). The angular region covered by the plot is approximately $10^{\circ} \times 10^{\circ}$. Labels from 18 to 23 refer to $70 \mathrm{GHz}$ horns, from 24 to 26 refer to $44 \mathrm{GHz}$ horns, and 27 and 28 refer to $30 \mathrm{GHz}$ horns. The scan direction, orthogonal to the focal plane symmetry axis, is indicated by an arrow. All the relative angles in pairs of feeds aligned along the scan direction are shifted by $45^{\circ}$, with the exception of RCA24.

interface is provided by a structural ring connected to the HFI by six insulating struts locked to the LFI main frame through a shaped flange. The design of the interface ring allows HFI integration inside the LFI, as well as waveguide paths, while ensuring accurate alignment of the $4 \mathrm{~K}$ reference loads with the reference horns mounted on the FEMs.

\subsubsection{Feed horns}

The LFI feeds must have highly symmetric beams, low levels of side lobes $(-35 \mathrm{~dB})$, cross-polarisation $(-30 \mathrm{~dB})$, and return loss $(-25 \mathrm{~dB})$, as well as good control of the phase centre location (Villa et al. 2002). Dual profiled conical corrugated horns have been designed to meet these requirements, a solution that has the added advantage of high compactness and design flexibility. The profiles have a sine squared inner section, i.e., $R(z) \propto \sin ^{2}(z)$, and by an exponential outer section, $R(z) \propto \exp (z)$.

The detailed electromagnetic designs of the feeds were developed based on the entire optical configuration of the feedtelescope system. The control of the edge taper only required minimal changes on the feed aperture and overall feed sizes, so that an iterative design process could be carried out at system level.

The evaluation of straylight effects in the optimisation process required extensive simulations (carried out with GRASP8 software) of the feed-telescope assembly for several different feed designs, edge tapers, and representative positions in the focal plane (Sandri et al. 2010). A multi-GTD (geometrical theory of diffraction) approach was necessary since the effect of shields and multiple scatter needed to be included in the simulations. In Table 4 we report the main requirements and characteristics of the LFI feed horns. The details of the design, 
Table 4. Specifications of the feed horns.

\begin{tabular}{lccc}
\hline \hline & $30 \mathrm{GHz}$ & $44 \mathrm{GHz}$ & $70 \mathrm{GHz}$ \\
\hline Band $[\mathrm{GHz}] \ldots \ldots \ldots \ldots \ldots \ldots \ldots$ & $27-33$ & $39.6-48.4$ & $63-77$ \\
Return loss $[\mathrm{dB}] \ldots \ldots \ldots \ldots \ldots \ldots$ & $<-25$ & $<-25$ & $<-25$ \\
Insertion loss at $20 \mathrm{~K}[\mathrm{~dB}] \ldots \ldots \ldots$ & -0.1 & -0.1 & -0.1 \\
Edge taper at $22^{\circ}[\mathrm{dB}] \ldots \ldots \ldots \ldots$ & 30 & 30 & 25 \\
Sidelobes $[\mathrm{dB}] \ldots \ldots \ldots \ldots \ldots \ldots$ & $<-35$ & $<-35$ & $<-35$ \\
Cross polarisation $[\mathrm{dB}] \ldots \ldots \ldots \ldots$ & $<-30$ & $<-30$ & $<-30$ \\
\hline
\end{tabular}

Table 5. Specifications of the OMTs.

\begin{tabular}{lccc}
\hline \hline & $30 \mathrm{GHz}$ & $44 \mathrm{GHz}$ & $70 \mathrm{GHz}$ \\
\hline Band $[\mathrm{GHz}] \ldots \ldots \ldots \ldots \ldots \ldots \ldots \ldots$ & $27-33$ & $39.6-48.4$ & $63-77$ \\
Isolation $[\mathrm{dB}] \ldots \ldots \ldots \ldots \ldots \ldots \ldots$ & $<-40$ & $<-40$ & $<-40$ \\
Return loss $[\mathrm{dB}] \ldots \ldots \ldots \ldots \ldots \ldots$ & $<-20$ & $<-20$ & $<-20$ \\
Insertion loss at $20 \mathrm{~K}[\mathrm{~dB}] \ldots \ldots \ldots$ & -0.15 & -0.15 & -0.15 \\
Cross polarisation $[\mathrm{dB}] \ldots \ldots \ldots \ldots$ & $<-25$ & $<-20$ & $<-20$ \\
\hline
\end{tabular}

manufacturing, and testing of the LFI feed horns are discussed in Villa et al. (2009b).

\subsubsection{Orthomode transducers}

The use of orthomode transducers (OMTs) allows the full power intercepted by the feed horns to be used by the LFI radiometers, and makes each receiver intrinsically sensitive to linear polarisation. The OMT splits the $\mathrm{TE}_{11}$ propagation mode from the output circular waveguide of the feed horn into two orthogonal polarised components. Low insertion loss $(<0.15 \mathrm{~dB})$ is needed to minimise any impact on radiometer sensitivity. In addition, OMTs are critical components for achieving the ambitious wide bandwidth specification, especially when combined with the miniaturisation imposed by the focal plane arrangement.

These requirements made commercial OMTs inadequate for LFI, and a dedicated design development was carried out for these components (Villa et al. 2009a). An asymmetric design was selected, with a common polarisation section connected to the feed horn and to the main and side arms in which the two polarisations are separated. A modular design approach was developed, in which six different sections were identified, each corresponding to a specific electromagnetic function. While the basic configuration of the OMTs at different frequencies is scaled from a common design, some details are optimised depending on frequency, such as the location of the waveguide twist in the side arm or in the main arm. The main design specifications for the LFI orthomode transducers are given in Table 5. Detailed discussion of the design, manufacturing, and unit-level testing of the LFI OMTs are reported in Villa et al. (2009a).

\subsubsection{Front-end modules}

The performance of the LFI relies largely on its front-end modules (FEMs, see Table 6). Detailed descriptions of the $30 \mathrm{GHz}$ and $44 \mathrm{GHz}$ FEMs are given by Davis et al. (2009), and of the $70 \mathrm{GHz}$ FEMs by Varis et al. (2009). Each FEM accepts four input signals, two from the rectangular waveguide outputs of the OMT and two from the $4 \mathrm{~K}$ reference loads viewed by rectangular horns attached to the FEMs (Fig. 6).

In each half-FEM, the sky and reference input signals were connected to a hybrid coupler (or "magic-T"). To minimise front-end losses, waveguide couplers were used, machined in the aluminium-alloy FEM body and gold-plated. At $20 \mathrm{~K}$ the hybrid losses were estimated 0.1 to $0.2 \mathrm{~dB}$. The first hybrid divides the signals between two waveguide outputs along which the low noise amplifiers (LNAs) and phase shifters were mounted. The internal waveguide design ensured that the phase is preserved at the input of the second hybrid. The signals were thus recombined at the FEM outputs as voltages that are proportional to the sky or reference load signal amplitude, depending on the state of the modulated phase switches.

At $70 \mathrm{GHz}$ the large number of channels called for a highly modular FEM design, where each half-module can be easily dismounted and replaced. In addition, the elements hosting the LNAs and the phase shifters (the so-called amplifier chain assembly, ACA, see Fig. 1) are built as separable units. This allowed flexibility during selection and testing, which proved extremely useful when replacement with a spare unit was required in an advanced stage of integration (Mennella et al. 2010). At 30 and $44 \mathrm{GHz}$, a multi-splitblock solution was devised to facilitate testing and integration, in which the four ACAs were mounted to end plates and arranged in a mirror-image format.

LNAs. To meet LFI requirements it was necessary to reach lower amplifier noise temperatures than previously achieved with multi-stage transistor amplifiers. We implemented state-ofthe art cryogenic InP HEMT technology at all frequencies. Each front-end LNA must have a minimum of $30 \mathrm{~dB}$ of gain to reject back-end noise, which required 4 to 5 stage amplifiers. In addition to low noise, the InP technology enables very low-power operation, which is essential for meeting the requirements for heat load at $20 \mathrm{~K}$. The amplifiers were selected and tuned for best operation at low drain voltages and for gain and phase match between paired radiometer legs, which is crucial for good balance.

The $70 \mathrm{GHz}$ receivers were based on monolithic microwave integrated circuit (MMIC) semiconductors (Fig. 14) while discrete HEMTs on a substrate (MIC technology) were used at 30 and $44 \mathrm{GHz}$. To reach lowest possible noise temperatures, ultra-short gate devices are adopted. The final design used $0.1 \mu \mathrm{m}$ gate length InP HEMTs manufactured by TRW (now Northrop Grumman) from the Cryogenic HEMT Optimisation Programme (CHOP).

Phase shifters. After amplification, the LNA output signals are applied to two identical phase shifters whose state is set by a digital control line modulated at $4 \mathrm{kHz}$. The signal is then conveyed via stripline to waveguide transitions to the second hybrid, and then passes to the interface with the interconnecting waveguide assembly to the BEMs. The phase switch design used at all frequencies is based on a double hybrid-ring configuration (Hoyland 2003). The switches, manufactured with InP PIN diodes, have demonstrated excellent cryogenic performances for low $1 / f$ noise contribution and good $180^{\circ}$ phase shift capability and amplitude balance.

Electrical connections. Each of the 11 FEMs uses 16 to 20 low noise transistors and 8 phase-switch diodes, all operated in cryo conditions. This sets demanding requirements in the design of the bias circuitry. The LNA biases are controlled by the data acquisition electronics (DAE) to obtain the required amplification and lowest noise operation. Different details in the design of the FEMs at each frequency minimise the number of supply lines. In the 30 and $44 \mathrm{GHz}$ FEMs, potentiometers were used to simplify the control wiring. The cryo-harness wiring that connects the FEMs to the power supply is $\sim 1.5 \mathrm{~m}$ in length (see Sect. 6.1), 
Table 6. Specifications of the front-end modules.

\begin{tabular}{|c|c|c|c|}
\hline & $30 \mathrm{GHz}$ & $44 \mathrm{GHz}$ & $70 \mathrm{GHz}$ \\
\hline Band $[\mathrm{GHz}] \ldots \ldots \ldots \ldots \ldots$ & $27-33$ & $39.6-48.4$ & $63-77$ \\
\hline Noise temperature over band $[\mathrm{K}]$ & 8.6 & 14.1 & 25.7 \\
\hline Gain (average over band) $[\mathrm{dB}]$.. & $30-33$ & $30-33$ & $30-33$ \\
\hline Gain variation with physical temperature $[\mathrm{dB} / \mathrm{K}] \ldots \ldots \ldots$. & 0.05 & 0.05 & 0.05 \\
\hline Noise temperature variation with physical temperature $[\mathrm{K} / \mathrm{K}]$ & 0.8 & 0.8 & 0.8 \\
\hline $1 / f$ knee frequency $[\mathrm{mHz}] \ldots \ldots \ldots \ldots \ldots \ldots \ldots \ldots$ & $<20$ & $<20$ & $<20$ \\
\hline Cross polarisation $[\mathrm{dB}] \ldots$ & $<-35$ & $<-35$ & $<-35$ \\
\hline Average power dissipation per FEM $[\mathrm{mW}] \ldots \ldots \ldots \ldots \ldots \ldots \ldots$ & 31 & 31 & 24 \\
\hline
\end{tabular}

which requires each wire to be terminated in the FEM with electrical protection devices to avoid sharp spikes.

Proper tuning of the LNAs is critical for best performance. The front-end InP LNAs contain 4 stages of amplification at 30 and $70 \mathrm{GHz}$ and 5 stages at $44 \mathrm{GHz}$. The LNAs are driven by three voltages: a common drain voltage $\left(V_{\mathrm{d}}\right)$, a gate voltage for the first stage $\left(V_{\mathrm{g}_{1}}\right)$, and a common gate voltage for the remaining stages $\left(V_{\mathrm{g}_{2}}\right)$ (Fig. 15). The voltages $V_{\mathrm{g}_{1}}$ and $V_{\mathrm{g}_{2}}$ are programmable and are optimised in the tuning phase (Cuttaia et al. 2009). The total drain current, $I_{\mathrm{d}}$ flowing in the ACA is measured and is available in the instrument housekeeping.

\subsection{The $4 \mathrm{~K}$ reference load system}

Blackbody loads provide stable internal signals for the pseudocorrelation receivers (Valenziano et al. 2009). Cooling the loads as close as possible to the $\sim 3 \mathrm{~K}$ sky temperature minimises the radiometer knee frequency (Eq. (16)) and reduces the susceptibility to thermal fluctuations and to other systematic effects. Requirements are thus derived for the loads' absolute temperature, $T_{4 \mathrm{~K}}<5 \mathrm{~K}$, as well as for the insertion loss of the reference horn, $L_{4 \mathrm{~K}}<0.15 \mathrm{~dB}$.

Connecting the loads to the HFI $4 \mathrm{~K}$ stage imposes challenging thermo-mechanical requirements on the system. The loads must be thermally isolated from, but radiometrically matched to, the reference horns feeding the $20 \mathrm{~K}$ FEMs. Complete thermal decoupling is obtained by leaving a $\sim 1.5 \mathrm{~mm}$ gap between the loads and the reference horns.

Radiometric requirements for the loads were derived by analysis and tests (Valenziano et al. 2009). The coupling between the reference horns and the loads is optimized to reduce reflectivity and to avoid straylight radiation leaking through the gap, with a required match at the horn aperture $>20 \mathrm{~dB}$. For the $70 \mathrm{GHz}$ radiometers the reference horns are machined as part of the FEM body, while for the 30 and $44 \mathrm{GHz}$ they are fabricated as independent waveguide components and mounted on the FEM (Fig. 12). This is because of the different paths required to reach the loads, whose location on the $4 \mathrm{~K}$ box is constrained by the LFI-HFI interface.

The configuration of the loads is the result of a complex trade-off between RF performance and a number of constraints such as allowed mass, acceptable thermal load on the $4 \mathrm{~K}$ stage, and available volume. The latter is limited by the optical requirement of placing the sky horns close to the focal plane centre. In addition, the precise location and alignment of the loads on the HFI box need to follow the non-trivial orientation and arrangement of the FEMs, dictated by the optical requirements for angular resolution and polarisation. The final design comprises a front layer (made in ECR-110) shaped for optimal match with the reference horn radiation pattern and a back layer (ECR-117) providing excellent absorption efficiency.

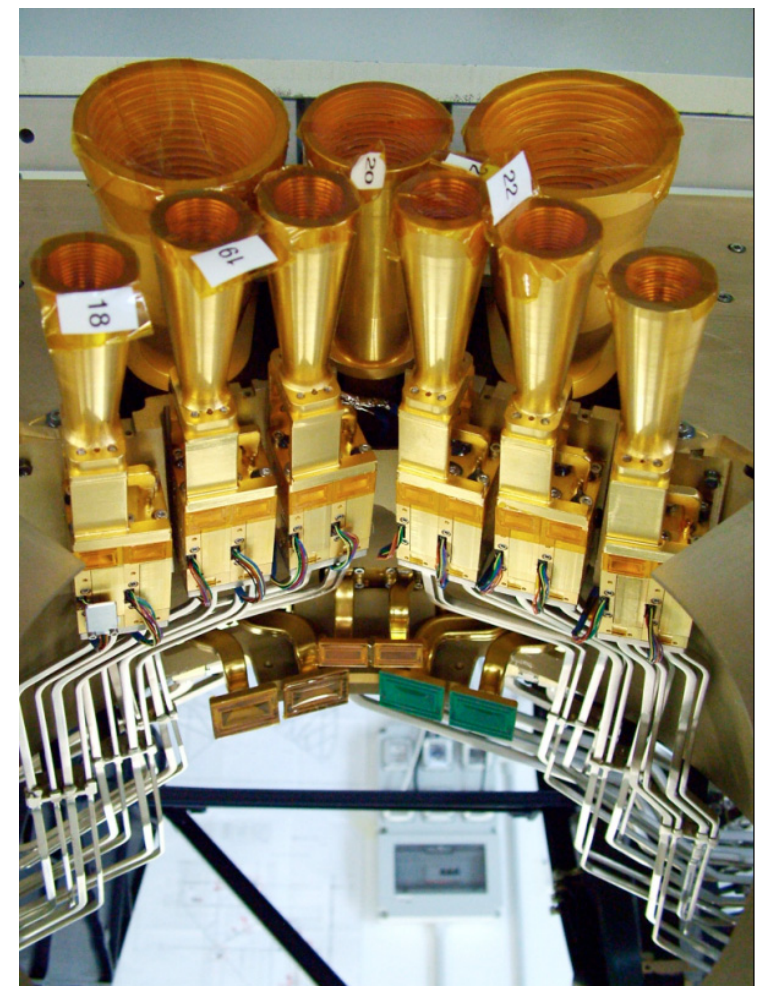

Fig. 12. Picture of nine of the eleven feed horns and associated OMTs, FEMs, and waveguides before integration of the HFI front end. The six smaller feeds in the front supply the $70 \mathrm{GHz}$ channels. In the back row, the two $30 \mathrm{GHz}$ feeds flank one of the $44 \mathrm{GHz}$ feeds. Visible are part of the twisted $\mathrm{Cu}$ waveguide sections and the reference horns (protected by a kapton layer): for the $70 \mathrm{GHz}$ FEMs they are embodied in the FEMs, while for the 30 and $44 \mathrm{GHz}$ they are flared waveguide sections.

Requirements on thermal stability at the $4 \mathrm{~K}$ shield interface were set to $10 \mu \mathrm{K} \sqrt{s}$, i.e., at the same level as the HFI internal requirement. To maximise thermal stability, a PID (proportional, integral, and derivative) system was implemented on the HFI $4 \mathrm{~K}$ box (Lamarre et al. 2010). The $70 \mathrm{GHz}$ loads are located near the top of the HFI $4 \mathrm{~K}$ box, near the PID control system, while the 30 and $44 \mathrm{GHz}$ loads are in the lower part (Figs. 12 and 16). This resulted in a more stable signal for the LFI $70 \mathrm{GHz}$ loads than for the 30 and $44 \mathrm{GHz}$ ones. Simulations have shown that residual systematic effects on the maps at end-of-mission, after applying destriping algorithms (Keihänen et al. 2004), are expected to be $\lesssim 1.5 \mu \mathrm{K}$ at $30-44 \mathrm{GHz}$ and $\lesssim 0.2 \mu \mathrm{K}$ at $70 \mathrm{GHz}$.

\subsection{Waveguides}

A total of 44 waveguides connect the $20 \mathrm{~K}$ FEU and the $300 \mathrm{~K}$ BEU through a length of 1.5 to $1.9 \mathrm{~m}$, depending on RCA. 

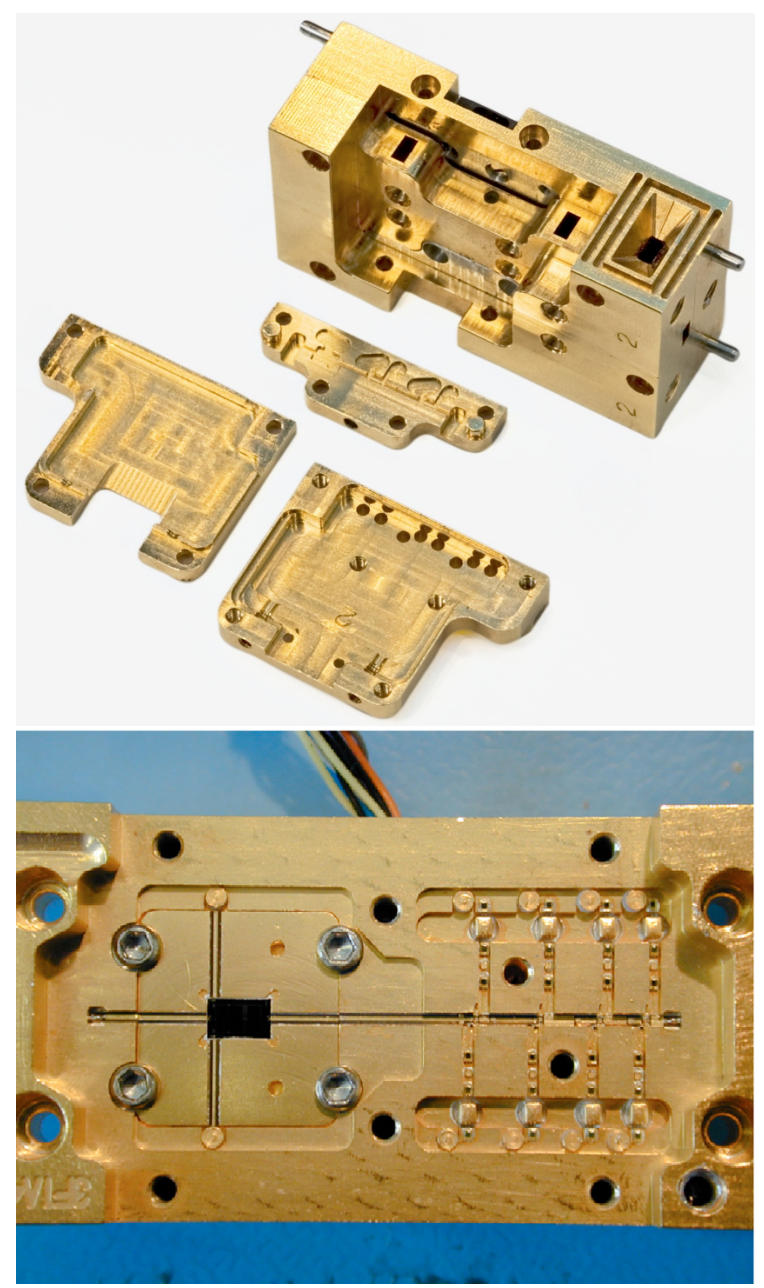

Fig. 13. Top: structure of a $70 \mathrm{GHz}$ half-FEM. On the right side of the module, one can see the small reference horn used to couple to the $4 \mathrm{~K}$ reference load surrounded by quarter-wave grooves. The parts supporting the amplifier chain assembly are dismounted and shown in the front. Bottom: picture of LNA and phase switch within a $30 \mathrm{GHz}$ FEM. The RF channel incorporating the four transistors runs horizontally in this view. The phase switch is the black rectangular element on the left.

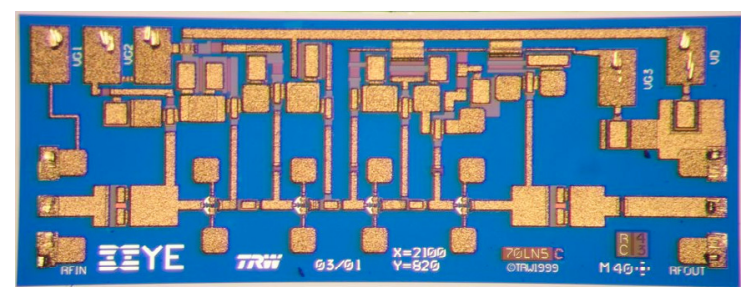

Fig. 14. A 4-stage InP HEMT MMIC low-noise amplifier used in a flight model FEM at $70 \mathrm{GHz}$. The size of the MMIC is $2.1 \mathrm{~mm} \times 0.8 \mathrm{~mm}$.

Conflicting constraints of thermal, electromagnetic, and mechanical nature imposed challenging trade-offs in the design. The LFI waveguides must ensure good thermal isolation between the FEM and the BEM, while avoiding excessive attenuation of the signal. In addition, their mechanical structure must comply with the launch vibration loads. The asymmetric location of the FEMs in the focal plane and the need to ensure integrability of the HFI in the LFI main frame, as well as of the RAA on the spacecraft, impose complex routing with several

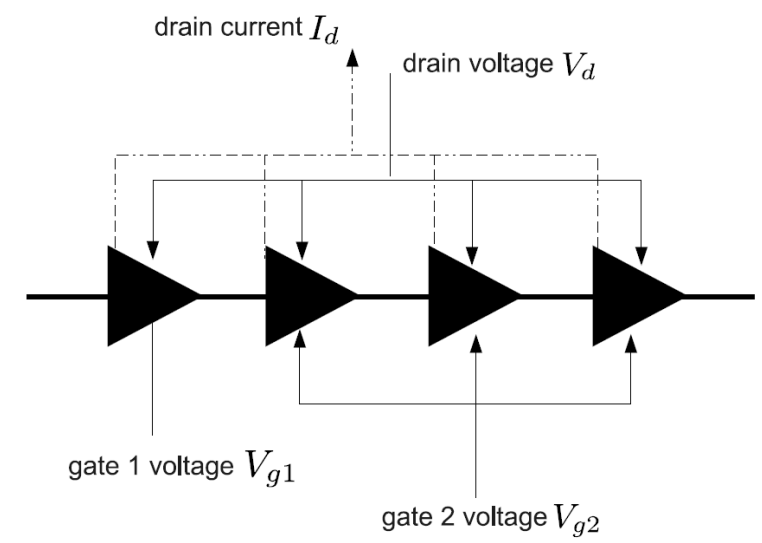

Fig. 15. DAE biasing to a front-end LNA. Each LNA is composed of four to five amplifier stages driven by a common drain voltage, a dedicated gate voltage to the first stage (most critical for noise performances), and a common gate voltage to the other stages.

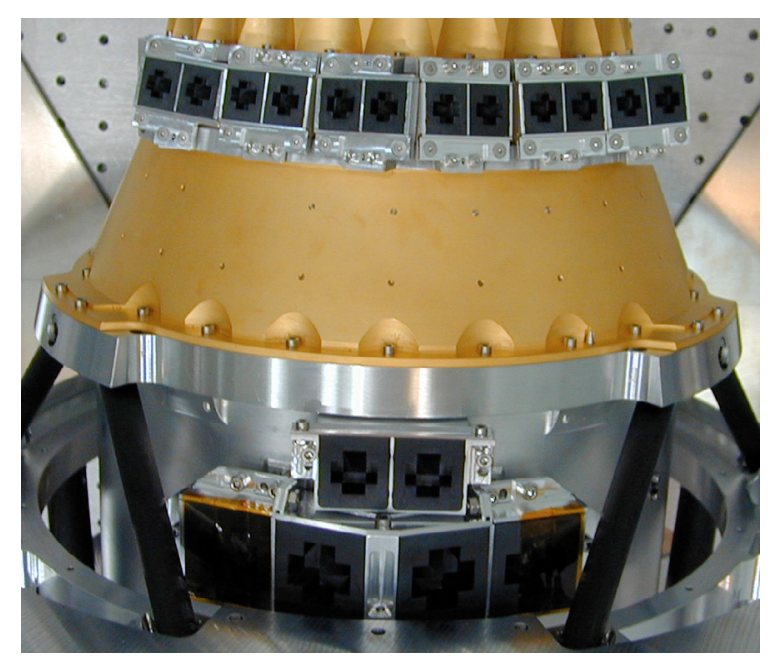

Fig. 16. Picture of the reference loads mounted on the HFI $4 \mathrm{~K}$ box. On the top is the series of loads serving the $70 \mathrm{GHz}$ radiometers, while in the lower portion are the loads of the two $30 \mathrm{GHz}$ FEMs and one of the $44 \mathrm{GHz}$ FEMs. Each FEM is associated to two loads, each feeding a radiometer in the RCA.

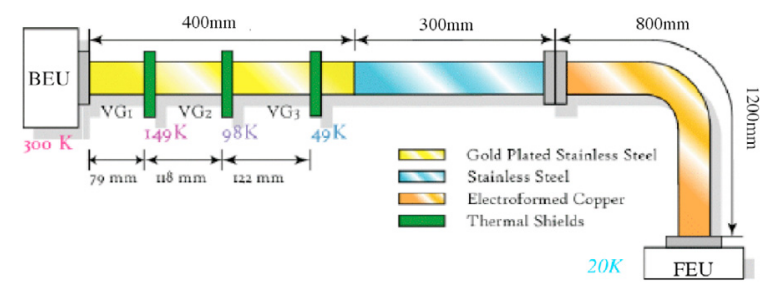

Fig. 17. Schematics of the LFI composite waveguide design, showing representative dimensions of the various sections as described in the text.

twists and bends, which required a dedicated design for each individual waveguide.

A composite configuration was devised with two separated sections: a stainless steel (SS) straight section connecting to the BEMs, and a copper $(\mathrm{Cu})$ section incorporating all the twists and bends and connecting to the FEMs (Fig. 17). The two sections are connected via custom-designed multiple flanges, each serving the four guides in each RCA.

The SS sections essentially support the entire 20-300 K thermal gradient. All 44 SS waveguides have the same length 
Table 7. RF requirements on the waveguides.

\begin{tabular}{|c|c|c|c|}
\hline & $30 \mathrm{GHz}$ & $44 \mathrm{GHz}$ & $70 \mathrm{GHz}$ \\
\hline Insertion loss $[\mathrm{dB}] \ldots \ldots$ & -2.5 & -3 & -5 \\
\hline Return loss $[\mathrm{dB}] \ldots$ & $<25$ & $<25$ & $<25$ \\
\hline Isolation $[\mathrm{dB}] \ldots \ldots \ldots \ldots \ldots \ldots \ldots$ & $<30$ & $<30$ & $<30$ \\
\hline Waveguide size $\ldots \ldots \ldots \ldots \ldots \ldots \ldots$ & WR28 & WR22 & WR12 \\
\hline Waveguide internal section $[\mathrm{mm}] \ldots \ldots$ & $7.112 \times 3.556$ & $5.690 \times 2.845$ & $3.099 \times 1.549$ \\
\hline
\end{tabular}

(70 $\mathrm{cm})$ and are gold-plated $(2 \mu \mathrm{m}$ thickness $)$ in the first $40 \mathrm{~cm}$ near the BEM interface to minimise ohmic losses. They are thermally sunk to the three V-grooves (Fig. 18), and their outer surfaces are painted black (Aeroglaze Z306) to optimise heat radiation.

The $\mathrm{Cu}$ sections, of lengths 80 to $120 \mathrm{~cm}$ depending on RCA, operate at a nearly constant temperature of $20 \mathrm{~K}$, and are individually designed based on optimisation of return loss compatible with the required routing. Precise criteria for the curvature radii, twist length, and mechanical tolerances were followed in the design (D'Arcangelo et al. 2009). Dynamical analysis showed the need for two dedicated mechanical support structures to ensure compliance with the vibration loads of the Ariane 5 launch (Fig. 18).

The selected design proved to meet simultaneously the heat load limits to the $20 \mathrm{~K}$ stage $(250 \mathrm{~mW}$ for the bundle of 44 guides) and the insertion loss at a level of few dBs. A thermal model was developed to calculate the temperature profile along the waveguide and the final solution was found using an analytical model.

D'Arcangelo et al. (2009) gives a full account of the manufacturing, qualification, and challenging test plan of the LFI waveguides.

\subsection{Back-end unit}

\subsubsection{Back-end modules}

The back-end modules (BEMs) are housed in the LFI back-end unit, together with the DAE, and are operated at room temperature $\sim 300 \mathrm{~K}$. Each BEM has four branches, grouped in pairs (Fig. 6). In each channel within the BEM, the incoming signal is filtered by a band-pass filter, amplified by cascaded transistor amplifiers, detected by a detector diode, and DC-amplified. The BEM casing also incorporates bias and protection circuits and connectors. Room temperature noise figures $<3 \mathrm{~dB}$ and an overall amplification of 20 to $25 \mathrm{~dB}$, depending on RCA, are specified for the BEM channels. Amplifier and detector diode instabilities are efficiently removed by the $4 \mathrm{kHz}$ phase switching, so that amplifier knee frequencies of $\sim 100 \mathrm{~Hz}$ are acceptable.

Amplifiers. The 30 and $44 \mathrm{GHz}$ BEMs (Artal et al. 2009) use MMIC gallium arsenide (GaAs) amplifiers. Each LNA consists of two cascaded stages. The $30 \mathrm{GHz}$ MMICs are commercial circuits using four stages of pseudomorphic HEMTs with an operating bandwidth from 24 to $36 \mathrm{GHz}, 23 \mathrm{~dB}$ of gain, and a $3 \mathrm{~dB}$ noise figure. The $44 \mathrm{GHz}$ MMICs were manufactured with a process employing a $0.2 \mu \mathrm{m}$ gate length P-HEMT on GaAs. The $70 \mathrm{GHz}$ BEMs (Varis et al. 2009) used the same type of InP MMIC amplifiers as in the FEMs. Although not required for performance or power dissipation constraints, this solution proved convenient in conjunction with the FEM development.
Band pass filters. Band pass filters in the BEMs were used to define the bandwidth and to reject out-of-band parasitic signals. In the 30 and $44 \mathrm{GHz}$ units the filter is based on a microstripcoupled line structure that inherently provides bandpass characteristics. Waveguide filters are used at $70 \mathrm{GHz}$, where the wavelength-scale cavity has an acceptable size.

Detector diodes The detector design at 30 and $44 \mathrm{GHz}$ uses commercially available GaAs planar doped-barrier Schottky diodes. The diodes were mounted with a coplanar-to-microstrip transition to facilitate on-wafer testing prior to integration in the BEM. As in the FEM design, at $70 \mathrm{GHz}$ the filters and the amplifier-detector assemblies of paired channels were mounted on separable modules to offer greater flexibility in the testing and optimisation phases.

DC amplifiers The detector diode is followed by a low-noise DC-amp with a voltage gain adequate to the required analogue output voltage range for the DAE interface. To meet EMC requirements and grounding integrity, the output voltages are provided as differential signals.

\subsubsection{Data acquisition electronics}

The acquisition and conditioning of the science signals from the BEMs and of housekeeping data is performed by the data acquisition electronics. The DAE also provides power supply, conditioning, and distribution to the RAA, in particular DC biasing to the LNAs and phase switches in the FEMs, as well as to the BEM amplifiers. The DAE tags the acquired data using the information of its on-board time to ensure that correlation can be made on the ground for proper pointing reconstruction. The raw data are then transmitted to the science processing unit for onboard processing.

As shown schematically in Fig. 9, the DAE functions are distributed into different sub-units. The "DAE-BEU box" and the "lateral trays" incorporate all the main functions, and are located in the back-end unit with the radiometer BEMs (Fig. 19). A separate "DAE power box" is interfaced with the spacecraft in order to receive the primary power supply and generate the needed secondary voltages to the DAE.

The DAE-BEU box is in charge of conditioning and acquiring the science data. The signals coming from the 44 detectors are integrated and held during the synchronous sampling and conversion. Science signals are digitised with 14-bit analogueto-digital converters using a successive approximation conversion algorithm. There are 44 independent analogue acquisition chains, one for each detector arm. To optimise the analogue signals from the BEMs to the ADC dynamic range, dedicated circuits remove an offset and then amplify the DC signal. Both offset and gain are programmable and are optimised as part of the instrument calibration process (Cuttaia et al. 2009; Mennella et al. 2010). In the optimised configuration, the number of counts 

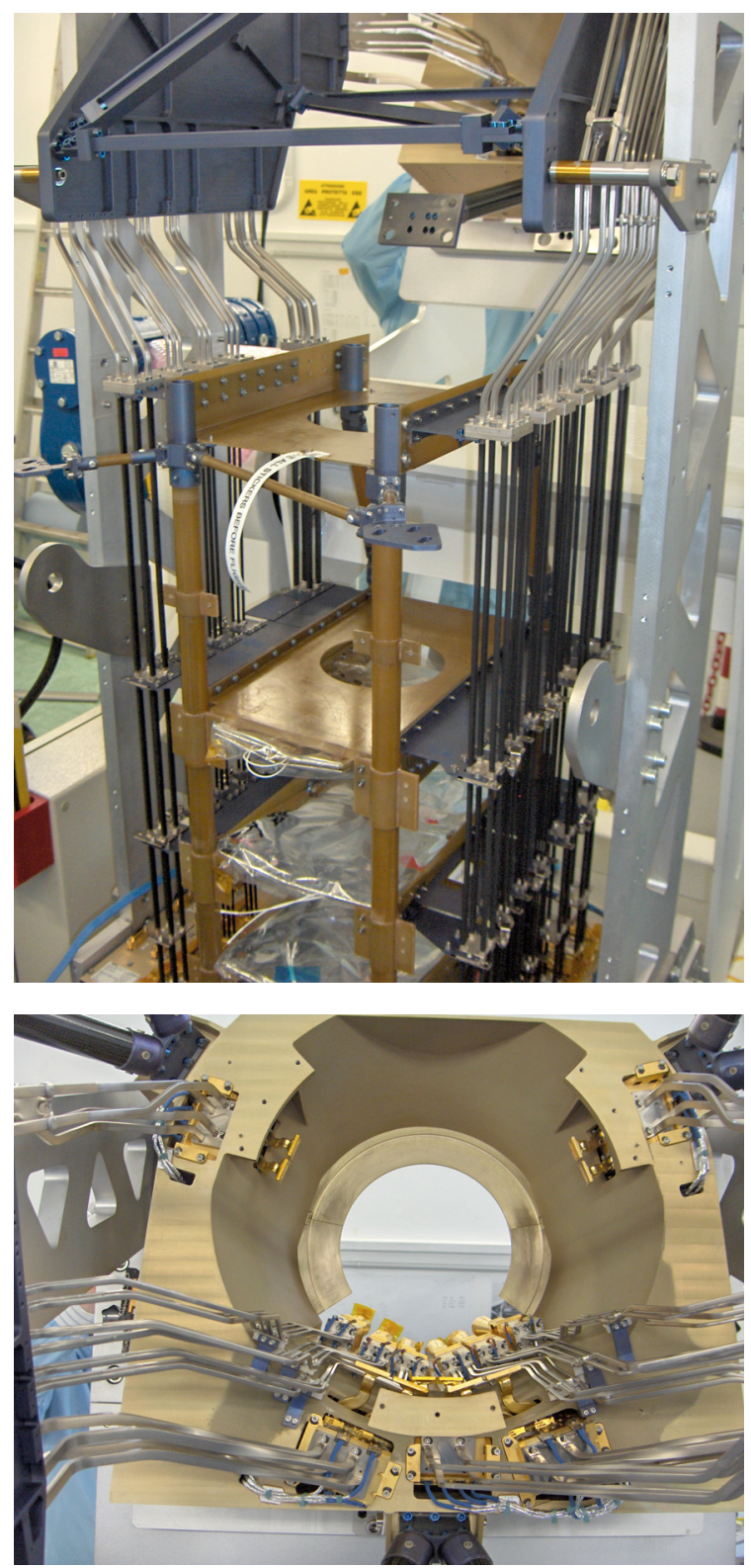

Fig. 18. Pictures of the LFI waveguides mounted on the RAA during the integration of the LFI flight model. Top: a view of the straight SS sections, black-painted on the outside and arranged in groups of four. On the upper part the $\mathrm{Cu}$ sections are connected through multiple flanges. The upper and lower mechanical support structures are also visible. The three interface levels corresponding to the three V-grooves are also shown. Bottom: back view of the LFI front-end unit showing the twisted $\mathrm{Cu}$ sections connecting to the FEMs. The waveguide routing and central hole in the main frame are designed to interface with the HFI front-end $4 \mathrm{~K}$ box.

exercised by the radiometer noise varies from 10 to 450, depending on channel. Acquired data are converted into serial streams and automatically transferred to the signal-processing unit in the REBA through synchronous serial links for processing and compression (Sect. 4.5).

The DAE is also in charge of collecting and storing housekeeping data in a dedicated RAM. This information is retrieved by the REBA and organised into two dedicated packets with periods of 1 and $32 \mathrm{~s}$, depending on the needed monitoring frequency. Housekeeping parameters include current consumptions in the FEMs and temperature sensors, which are essential for

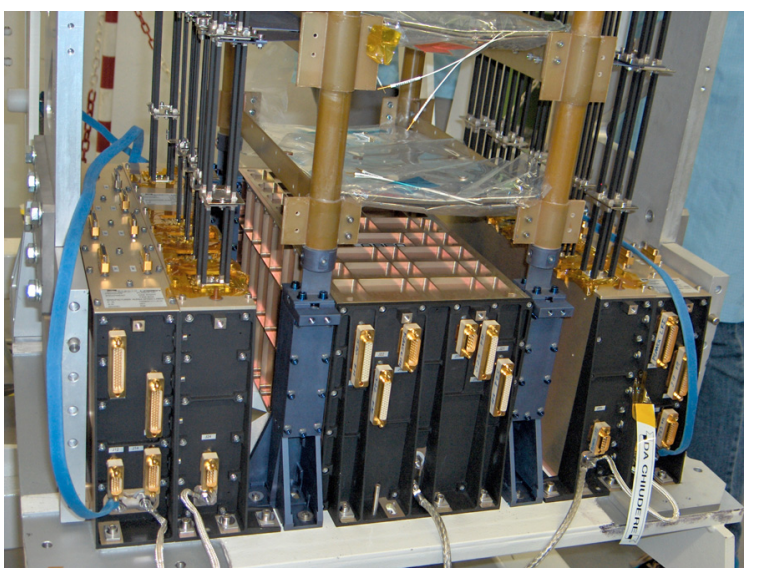

Fig. 19. Picture of the lower part of the LFI RAA during an advanced phase of the instrument flight-model integration showing the LFI backend unit. The two lateral trays hosting the radiometer BEMs are symmetrically disposed to the left and right sides of the DAE-BEU box. The lower part of the straight stainless steel waveguides are shown.

trend analysis and systematic error tests. These data are used extensively during the functionality checks of the radiometers.

The two DAE "lateral trays" contain the circuitry needed to provide the power supply to the RCAs, divided into four power groups (Fig. 9). These power supplies are independent of each other to minimise crosstalk and interference. The bias of each FEM and the controls for the phase switches are regulated to selectable voltage levels and filtered to achieve a minimum level of conducted noise. Particularly critical for the instrument performance is the optimal biasing of the FEM amplifiers. As described in Sect. 4.1.5, the bias voltages of the first LNA stage and of the following stages are programmable separately.

\subsection{Radiometer electronics box (REBA)}

Downstream of the DAE, the LFI signals are digitally processed by the REBA (radiometer electronics box assembly), which also contains the power supply for LFI and the interface with the satellite SVM. The electronics hardware and on-board software are discussed by (Herreros et al. 2009). The REBA is a fully redundant unit, and it is internally separated into different subunits as shown schematically in Fig. 9.

The signal processing unit (SPU) receives the raw digital science data from the DAE and performs on-board signal averaging, data compression, and science telemetry packetisation. The need to reject $1 / f$ noise led to raw data sampling at $8192 \mathrm{~Hz}$ ( $122 \mu \mathrm{s} /$ sample), the LFI internal clock generator frequency. The clock synchronously drives the phase switches in the FEMs, the ADCs, and the on-board processor, which reconstructs the ordering of the acquired signals and synchronises it with the onboard time. Taking housekeeping and ancillary information into account, this corresponds to a data rate of $\sim 5.7 \mathrm{Mbps}$, or a factor of 100 higher than the allocated data rate for the instrument, $53.5 \mathrm{Kbps}$. Averaging the samples from sky and reference-load signals to within the Nyquist rate on the sky (3 bins per HPBW at each frequency) drastically reduces the data volume, leaving a compression requirement of a factor 2.4 (see Sect. 6). The adopted algorithm implemented in the SPU relies on threestep processing of nearly loss-less compression that requires 5-parameter tuning to be optimised. The details of the LFI data compression strategy and end-to-end test results are discussed by Maris et al. (2009). 
The main functions of the data processing unit (DPU) include monitoring and control of the RAA, instrument initialisation, error management, on-board time synchronisation, management of instrument operating modes, and control of the overall LFI data rate and data volume. Switching the FEMs and BEMs on and off, as well as voltage adjustments, are addressed by the DPU with a configuration that allows flexible setup commands. The DPU interface provides all commands for the DAE, while the SPU interface is in charge of retrieving the fixed format raw data from the RCAs. Both the DPU and the SPU are based on an $18 \mathrm{MHz}$ CPU. The link between the REBA and the DAE is implemented through IEEE 1355 interfaces and by means of data flag signals that ensure hardware and software synchronisation.

Finally, the data acquisition unit (DAU) is in charge of functions that are internal to the REBA, and it has no interfaces with the RAA. It converts the primary power received from the spacecraft to the secondary regulated voltages required by the REBA and performs analogue-to-digital conversion of REBA housekeeping data.

\section{Thermal interfaces}

\subsection{LFI $20 \mathrm{~K}$ stage}

The LFI front-end is cooled to $20 \mathrm{~K}$ by a closed-cycle hydrogen sorption cryo-cooler (Wade et al. 2000; Bhandari et al. 2004; Morgante et al. 2009), which also provides $18 \mathrm{~K}$ pre-cooling to the HFI (Fig. 20). The cooler provides $\sim 1 \mathrm{~W}$ of cooling power for the LFI FEU. The system operates by thermally cycling a set of compressors filled with $\mathrm{La}_{1.0} \mathrm{Ni}_{4.78} \mathrm{Sn}_{0.22}$ powder alternately absorbing and desorbing $\mathrm{H}_{2}$ gas as their temperature is cycled between $\sim 270 \mathrm{~K}$ and $\sim 450 \mathrm{~K}$, thus providing the working fluid in a Joule-Thomson (JT) refrigerator.

Heating of the sorbent beds is obtained by electrical resistance heaters, while cooling is achieved by thermally connecting the compressor element to a radiator at $\sim 270 \mathrm{~K}$ in the warm spacecraft. The hydrogen flow lines are connected to the three $\mathrm{V}$-groove radiators and passively pre-cooled to $<50 \mathrm{~K}$ before reaching the $20 \mathrm{~K} \mathrm{JT}$ expansion valve.

In the complete system, six identical compressors are used, while a high-capacity storage sorbent bed is used as a gas reservoir in the low-pressure line. At any time, one compressor is hot and desorbing to provide high-pressure hydrogen gas in the range $30-50 \mathrm{~atm}$, one compressor is heating up, one is cooling down, while the other three are cold and absorbing gas at $\sim 0.25 \mathrm{~atm}$. This principle of operation ensures that no vibrations affect the detectors, a unique property of this kind of cooler, which is very beneficial to Planck.

As a consequence of the cooler cycles, the system exhibits two characteristic (controllable) time periods: $\tau_{\text {bed }}$, syncronous with each sorbent bed cycle (nominally $\tau_{\text {bed }}=667 \mathrm{~s}$ ), and $\tau_{\text {system }}=6 \times \tau_{\text {bed }}$, for the whole system cycle. Temperature fluctuations at the cold end are expected to modulate at these periods and may affect the LFI scientific performance both by direct coupling to the $20 \mathrm{~K} \mathrm{FPU}$ and by fluctuations induced in the $4 \mathrm{~K}$ reference loads through the pre-cooling interface with HFI at $18 \mathrm{~K}$. We analysed this source of systematic effects extensively in the design phase by propagating its effects to the map level (Mennella et al. 2002b). We derived stringent requirements $(\delta T<100 \mathrm{mK}$ peak-to-peak) on acceptable temperature fluctuations at the interfaces of the $20 \mathrm{~K}$ cooler with LFI (LVHX2). Testing at instrument level (Tomasi et al. 2010) and at system level have verified the design consistency.

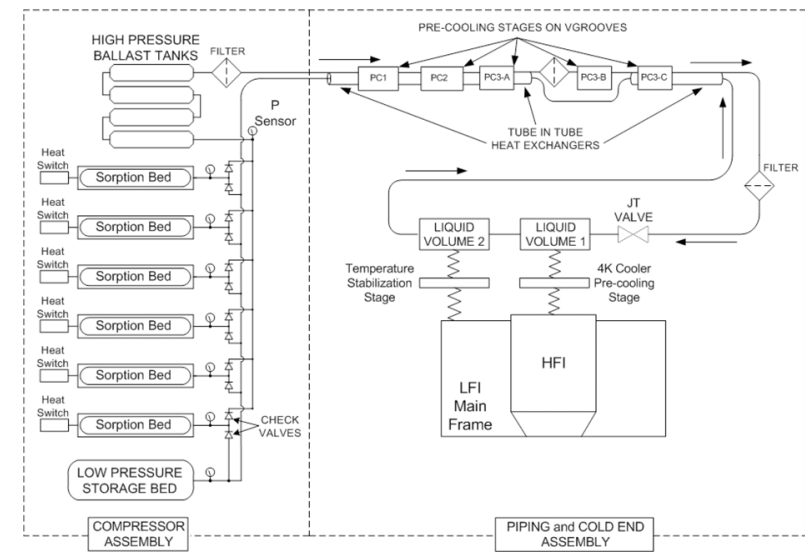

Fig. 20. Schematic of the $20 \mathrm{~K}$ sorption cooler. The compressor assembly, located in the Planck service module, is shown in the left and comprises the six sorption beds, the low pressure storage bed, the high pressure tanks. The pre-cooling stages on the V-grooves, the J-T valve and the cold-end interfaces with the LFI and HFI instruments are shown on the right.

Table 8. $20 \mathrm{~K}$ heat load budget.

\begin{tabular}{|c|c|}
\hline Item & $P[\mathrm{~mW}]$ \\
\hline FEMs & 299 \\
\hline Waveguides ......... & 249 \\
\hline Cryoharness ......... & 3 \\
\hline Struts $\ldots \ldots \ldots \ldots \ldots$ & 23 \\
\hline Radiant coupling & 2 \\
\hline TOTAL $\ldots \ldots \ldots \ldots \ldots$ & 576 \\
\hline
\end{tabular}

Table 9. LFI heat loads and allocations on V-grooves.

\begin{tabular}{|c|c|c|c|}
\hline & $\begin{array}{r}T \\
{[\mathrm{~K}]}\end{array}$ & $\begin{array}{r}P_{\text {estimated }} \\
{[\mathrm{mW}]}\end{array}$ & $\begin{array}{r}P_{\text {allocated }} \\
{[\mathrm{mW}]}\end{array}$ \\
\hline VG3 & 51.4 & 463 & 710 \\
\hline VG2 & 106.2 & 337 & 560 \\
\hline VG1 & 166.2 & 2939 & 5370 \\
\hline
\end{tabular}

\subsection{Thermal loads}

The limited cooling power of the sorption cooler imposes requirements on acceptable heat loads at $20 \mathrm{~K}$. This includes power dissipated by the amplifiers and phase switches in the front-end, as well as parasitic loads from the waveguides, cryoharness, and other passive elements. As discussed in Sect. 4 these were strong drivers in the architecture of the radiometer chains and in the design of the waveguides and cryo-harness (Sects. 4.3 and 6.1). Table 8 summarises the heat load budget at $20 \mathrm{~K}$ for the LFI elements. The $299 \mathrm{~mW}$ allocated to the frontend modules has been split in an average dissipation of $31 \mathrm{~mW}$ per FEM at 30 and $44 \mathrm{GHz}$, and $24 \mathrm{~mW}$ per FEM at $70 \mathrm{GHz}$.

As part of the system thermal design, upper limits to heat loads on each of the three V-grooves were allocated to the LFI. Table 9 shows the estimated loads from the LFI compared to the budget allocations, showing that compliance has been achieved with ample margins.

\subsection{Temperature sensors}

Temperature sensors are placed in strategic locations of the instrument flight model to monitor temperature values and 
Table 10. Main characteristics and specifications of the LFI cryoharness.

\begin{tabular}{|c|c|c|c|c|c|c|c|c|}
\hline \multirow{2}{*}{ ITEM } & \multicolumn{4}{|c|}{ REQUIREMENT } & \multicolumn{4}{|c|}{ DESIGN SOLUTION } \\
\hline & $N$ & $I_{\max } 20 \mathrm{~K}$ & $I_{\max } 300 \mathrm{~K}$ & $\begin{array}{l}R \\
0\end{array}$ & Material & Diameter & $\begin{array}{l}R \\
\Omega\end{array}$ & $P$ at FPU \\
\hline $\begin{array}{l}\text { ITEM } \\
\text { HEMT GND }\end{array}$ & $\frac{1 v}{11}$ & [1107] & $\frac{[\mathrm{mA}]}{200}$ & $<1$ & Copper & $\frac{[\mathrm{Aw}, \mathrm{mm}]}{38(0.1)}$ & $\begin{array}{l}32 \\
0.6\end{array}$ & 6.32 \\
\hline HEMT Drain & 44 & 10 & 50 & $<5$ & Nickel & $38(0.1)$ & 2.2 & 11.37 \\
\hline HEMT Gate ....... & 88 & 100 & 100 & $<200$ & Manganin & $40(0.08)$ & 193.4 & 1.86 \\
\hline Phase Switch GND . & 11 & 4 & 4 & $<10$ & Titanium & $32(0.2)$ & 5.2 & 1.55 \\
\hline Phase Switch ........ & 88 & 1 & 1 & $<50$ & Titanium & $38(0.1)$ & 21.0 & 5.88 \\
\hline Shield $\ldots \ldots \ldots \ldots \ldots$ & $\ldots$ & $\ldots$ & $\ldots$ & $\ldots$ & $\mathrm{Al}+$ Kapton & $100 \mathrm{~nm}$ & $\ldots$ & 2.28 \\
\hline Temperature Sensors ..... & 48 & 40 & 40 & $<200$ & Manganin & $40(0.08)$ & 139.3 & 0.93 \\
\hline TOTAL $\ldots . . . \ldots \ldots \ldots$ & 290 & & & & & & & 30.20 \\
\hline
\end{tabular}

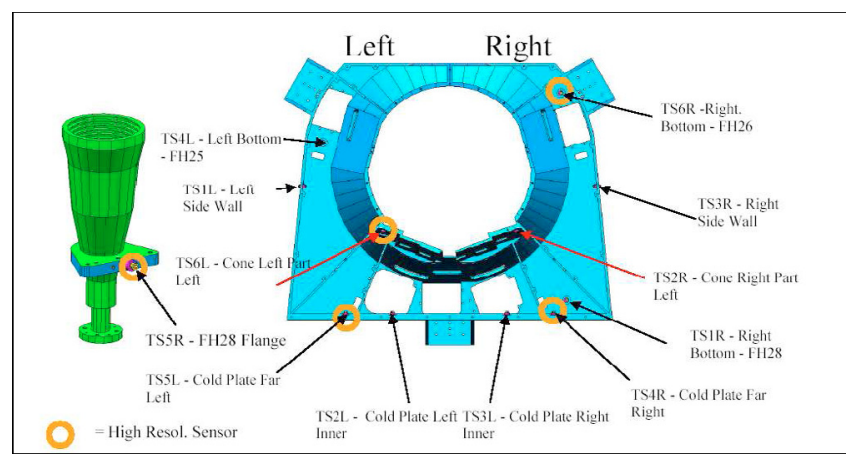

Fig. 21. Temperature sensors in the LFI front end unit. Circles indicate high-sensitivity sensors.

fluctuations during both ground calibration (Tomasi et al. 2010) and in-flight operation.

Figure 21 shows the 12 sensors in the focal plane unit, five of which have higher sensitivity and a narrower dynamic range ( 14 to $26.5 \mathrm{~K}$ ) to adequately trace temperature fluctuations. They are Lakeshore silicon diodes DT670, and the associated readout electronics leads to a typical sensitivity of $0.9 \mathrm{mK}$ at $20 \mathrm{~K}$. Averaging of multiple readings allows increasing the resolution below the quantization limit at the relevant fluctuation time scale. One of the sensors (TS5R) has been placed on the flange of a $30 \mathrm{GHz}$ feedhorn (RCA28) to directly monitor front-end stability. The interface to the sorption cooler cold end (LVHX2) is also monitored with sensors both on the LFI side and on the sorption cooler side. For analysis of in-flight data, additional temperature information will be used from sensors belonging to the HFI, the telescope, and the spacecraft.

\section{Electrical and communication interfaces}

\subsection{Cryo-harness}

Another challenging element in the LFI design is the electrical connection from the DAE power supply to the cryogenic frontend (cryoharness). Each FEM needs 22 bias lines for biasing the LNAs and phase switches. When including temperature sensor wires, a total of 290 lines have to be routed from the $300 \mathrm{~K}$ electronics to the $20 \mathrm{~K}$ FPU along a path of $\sim 2.2 \mathrm{~m}$. The lines need to transport currents ranging from a few $\mu \mathrm{A}$ (for temperature sensors) up to $200 \mathrm{~mA}$. The stability needed in the bias of the cryogenic LNAs calls for high immunity to external noise and disturbances, i.e., efficient electrical shielding. On the other hand, heat transport to $20 \mathrm{~K}$ needs to be kept at a few mW. Furthermore, to ensure operability of LFI at room temperature (a tremendous advantage in the integration and test process), the harness was required to be compatible with operation at $300 \mathrm{~K}$.

In Table 10 we show the main characteristics of the implemented cable design (Leutenegger et al. 2003), while Fig. 23 is the schematics of the cryoharness configuration and routing. The cryo-harness was mounted as part of the RAA and integrated before delivery, so that it could be kept integrated.

\subsection{Electromagnetic compatibility (EMC)}

Much effort has been made to ensure a highly stable electrical environment. The grounding scheme (Fig. 22) was optimised to ensure maximum protection of the bias lines to the frontend cryogenic LNAs and phase switches. Unwanted fast voltage transient across the input biases can damage the InP-based HEMT junctions; furthermore, the front-end module performance requires very low noise in the received bias.

The $20 \mathrm{~K}$ LFI focal plane is about $2 \mathrm{~m}$ away from the warm back-end unit, where the LNA biases are generated. Because InP technology requires the LNA substrates to be connected to the ground in order to avoid ground loops, the only grounding reference for the instrument is on the focal-plane, and the whole back-end electronics is referred to chassis at the radiometer's front-end.

The EMC design and verification approach was performed incrementally and based on analysis and testing at the component or subassembly level. Table 11 lists all the internal frequencies of the LFI instrument and sorption cooler. Whenever relevant, these were monitored in the test campaign as potential sources of RF disturbances, both within LFI and towards the HFI detectors. In fact, a critical aspect in the design was to ensure mutual compatibility between the two instruments, which are in mechanical contact at the FPU interface. This issue was analysed in detail by both instrument teams, however, a hardware verification was possible only at FM system level during the cryogenic performance test campaign performed at CSL in June-August 2008. The results confirmed excellent compatibility between the two instruments.

\subsection{Data rate}

The choice of an $\mathrm{L}_{2}$ orbit for Planck induces stringent requirements on the rate of data transmission to the ground. Both the sky and reference load samples will be transmitted to the ground, so that full data reduction can be performed at the LFI Data Processing Centre (DPC). After sample-averaging and data 
M. Bersanelli et al.: Planck pre-launch status: Design and description of the Low Frequency Instrument

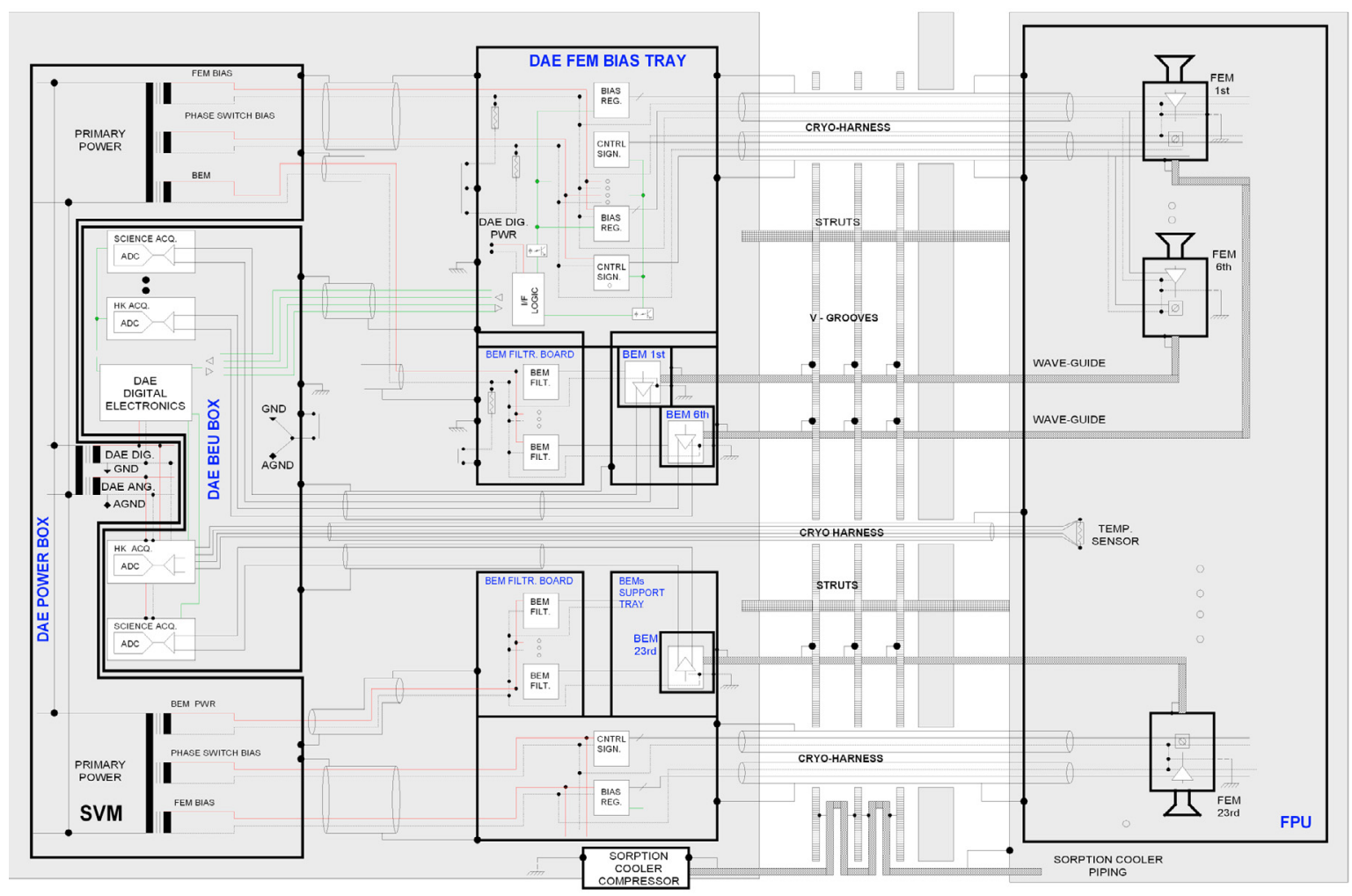

Fig. 22. Schematics of the grounding scheme of LFI.

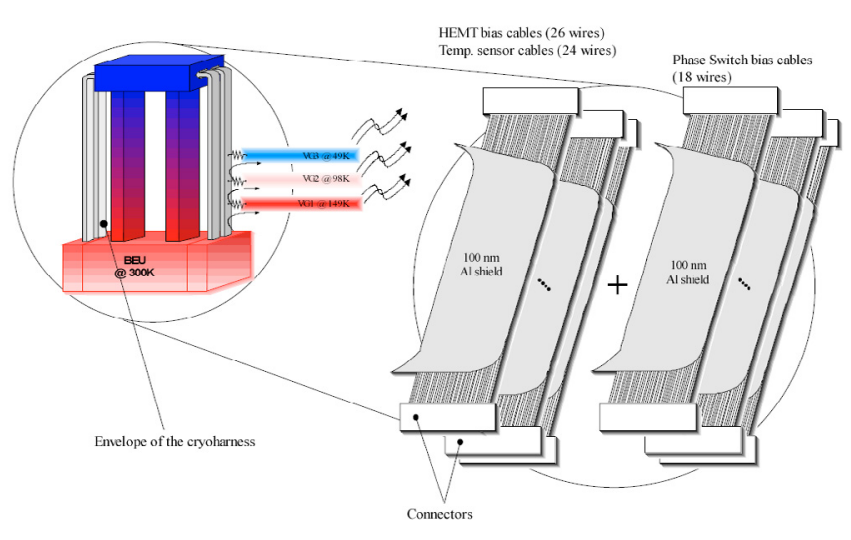

Fig. 23. Schematics of the cryoharness serving HEMT biasing, phase switch biasing, and temperature sensors. Heat loads on the $20 \mathrm{~K}$ stage are minimised by intercepting heat with the V-grooves.

compression (by a factor of 2.4 for at least the $95 \%$ of the packets) performed in the REBA SPU (Sect. 4.5), the science data volume is $36.12 \mathrm{Kbps}$, increased to $37.88 \mathrm{Kbps}$ by packeting overheads. An additional contribution of up to $5.06 \mathrm{Kbps}$ comes from the so-called "calibration channel": for diagnostic purposes, one LFI channel at a time will be transmitted to the ground without compression. Adding $2.57 \mathrm{Kbps}$ of housekeeping leads to a total budget of $45.41 \mathrm{Kbps}$ for LFI, well within the allocated $53.5 \mathrm{Kbps}$ (see Table 12). It is critical that the (average) 2.4 compression factor be achieved with an essentially lossless process, which requires careful optimisation of the parameters that control the on-board compression algorithm in the SPU (Maris et al. 2009). After telemetry transmission, the data will be treated through LFI DPC "Level 1" (Zacchei et al. 2009) for real-time assessment, housekeeping monitoring, and data decompression. Then the time-order information (TOI) will be
Table 11. LFI characteristic internal frequencies.

\begin{tabular}{lll}
\hline \hline$v$ & Origin & Unit \\
\hline $1 \mathrm{~Hz} \ldots \ldots \ldots$ & Housekeeping acquisition frequency & DAE BEU \\
$1 \mathrm{~Hz} \ldots \ldots \ldots \ldots$ & Synchronisation signal & DAE BEU, REBA \\
$10 \mathrm{~Hz} \ldots \ldots \ldots$ & SCS \\
$1 \mathrm{kHz} \ldots \ldots \ldots$ & Internal timer & SCS \\
$4096 \mathrm{~Hz} \ldots \ldots \ldots$ & Phase Switch & FEM, DAE BEU \\
$100 \mathrm{kHz} \ldots \ldots$ & $5 \mathrm{~V} \& 12 \mathrm{~V}$ DC/DC & SCS \\
$131072 \mathrm{~Hz} \ldots \ldots$ & DC/DC converters & DAE Power box \\
$131072 \mathrm{~Hz} \ldots \ldots$ & On-board clock signal & DAE BEU, REBA \\
$131072 \mathrm{~Hz} \ldots \ldots$ & LOBT clock & SCS \\
$200 \mathrm{kHz} \ldots \ldots$. & 12 V DC/DC & SCS \\
$1 \mathrm{MHz} \ldots \ldots \ldots$ & Command link from the BEU box & DAE power box \\
$1 \mathrm{MHz} \ldots \ldots \ldots$ & Internal transfer of digital data & DAE BEU, REBA \\
$8 \mathrm{MHz} \ldots \ldots \ldots$ & ADC clock & SCS \\
$10 / 80 \mathrm{MHz} \ldots \ldots$ & 1355 serial data digital interface & DAE BEU, REBA \\
$16 \mathrm{MHz} \ldots \ldots \ldots$ & DSP processor clock & SCS \\
$17.46 \mathrm{MHz} \ldots \ldots$ & Clock frequency of the DSP & REBA \\
$20 \mathrm{MHz} \ldots \ldots \ldots$ & Sequencer internal clock & DAE BEU \\
\hline
\end{tabular}

Table 12. LFI data rate summary.

\begin{tabular}{lcccc}
\hline \hline & $30 \mathrm{GHz}$ & $44 \mathrm{GHz}$ & $70 \mathrm{GHz}$ \\
\hline Number of detectors $\ldots \ldots \ldots \ldots \ldots \ldots \ldots$ & 8 & 12 & 24 \\
Angular resolution $($ nominal) & $\ldots \ldots \ldots$ & $33^{\prime}$ & $24^{\prime}$ & $14^{\prime}$ \\
Beam crossing time $[\mathrm{ms}] \ldots \ldots \ldots \ldots \ldots$ & 92 & 64 & 39 \\
Sampling rate $[\mathrm{Hz}] \ldots \ldots \ldots \ldots \ldots \ldots$ & 32.51 & 46.55 & 78.77 \\
Science data rate $[\mathrm{Kbps}] \ldots \ldots \ldots \ldots \ldots$ & 8.32 & 17.87 & 60.49 \\
\hline Total science data rate $\ldots \ldots \ldots \ldots \ldots \ldots$ & \multicolumn{5}{c}{$86.69 \mathrm{Kbps}$} \\
after compression $\ldots \ldots \ldots \ldots \ldots \ldots$ & \multicolumn{5}{c}{$46.12 \mathrm{Kbps}$} \\
Total LFI data rate $\ldots \ldots \ldots \ldots \ldots \ldots$ &
\end{tabular}

generated and processed by the successive analysis steps in the DPC pipeline. 
Table 13. Principal requirements and design solutions in LFI.

\begin{tabular}{ll}
\hline \hline Requirement/ & \\
Constraint & Design solution \\
\hline High sensitivity & $\begin{array}{l}\text { Cryogenically cooled }(\sim 20 \mathrm{~K}) \text { HEMT amplifiers. } \\
\text { Pow residual } 1 / f, \text { immunity from receiver systematics } \\
\text { Pseudo-correlation differential design. Cryogenic reference load }(\sim 4 \mathrm{~K}) . \text { Offset } \\
\text { removal by gain modulation factor in post-processing. Fast switching }(4 \mathrm{KHz}) \\
\text { of sky and reference signal to suppress backend } 1 / f \text { noise. }\end{array}$ \\
$\begin{array}{l}\text { "Internal" reference load. } \\
\text { Modularity, cryo testing. }\end{array}$ & $\begin{array}{l}\text { Phase switch in frontend modules. } \\
\text { Two amplification stages (cold frontend, warm backend). Low loss and thermal } \\
\text { conductivity interconnecting waveguides. } \\
\text { Low power dissipation at the } 20 \mathrm{~K} \text { stage. }\end{array}$ \\
$\begin{array}{l}\text { Phase switch and second hybrid in the frontend (avoids need of phase-matched } \\
\text { waveguides.) }\end{array}$ \\
\hline
\end{tabular}

\section{Optical interfaces}

The optimisation of the optical interface between the combined LFI-HFI focal plane and the Planck telescope was coordinated throughout the various development phases of the project. Rejection of systematic effects arising from non-ideal optical coupling has been a major design driver for LFI (Mandolesi et al. 2000b; Villa et al. 2009b). Minimisation of main beam ellipticity and distortion, particularly relevant for the off-axis LFI feeds, has been a key element in the optical design (Burigana et al. 1998; Sandri et al. 2010). An upper limit of $<1 \mu \mathrm{K}$ (rms) to straylight contamination from various sources was set as a design criterion. Far sidelobe effects were simulated for the Galactic foregrounds (diffuse dust, free-free, and synchrotron emission, and HII regions) (Burigana et al. 2001), as well as for solar system sources. The full beam pattern was calculated using a combination of physical optics (PO), physical theory of diffraction (PTD), and multi-reflector geometrical theory of diffraction (MrGTD) by considering radiation scattered by both reflectors, as well as reflection and diffraction effects on the baffle.

The final LFI optical design is discussed by Sandri et al. (2010) (see Maffei et al. 2010, for the analogous process for HFI). In particular, the LFI optical optimisation allowed design of the $70 \mathrm{GHz}$ feeds to meet straylight requirements and reach an angular resolution $\sim 13^{\prime}$ for most $70 \mathrm{GHz}$ channels, thus improving over the requirements value (14', Table 1$)$.

Emission originating within the Planck spacecraft and coupling directly into the LFI beams ("internal straylight") was also considered in the optical design as a potential source of systematic effect. An overall upper limit of $1 \mu \mathrm{K}$ was set for internal straylight and a breakdown of contributions from various optical elements of the payload module (baffle, reflectors structures, third V-groove) was carried out. Simulations have shown compliance with the allocated budget.

Knowledge of the microwave transmission of the LFI channels is an essential element for extracting polarisation information (Leahy et al. 2010) and for separating foreground components. The band shapes of each RCA channel have been evaluated from measurements at the single unit level, then combined with a dedicated software model, and finally verified with end-to-end testing as part of the RCA cryogenic test campaign (Zonca et al. 2009).

Alignment requirements on the FPU relative to the telescope were developed taking the thermo-elastic effects of the cooldown to $50 \mathrm{~K}$ of the LFI struts into account. The driving requirements were set by the HFI optical alignment, which were more stringent because of the shorter wavelengths. For the LFI, the internal alignment requirements between FEU and BEU of $\pm 2 \mathrm{~mm}$ required careful design of the waveguide support structures.

\section{Conclusions}

The Planck scientific objectives call for full-sky maps with sensitivity $\Delta T / T \simeq 2 \times 10^{-6}$ per $\Delta \theta \simeq 10^{\prime}$ pixel. The combination of LFI and HFI covers the spectral range 30 to $850 \mathrm{GHz}$, to allow precise removal of non-cosmological emissions. The two instruments use widely different technologies and will be affected differently by different sources of systematic effects. This unique feature of Planck provides a powerful tool for identifying and removing systematic effects. The Planck-LFI covers three frequency bands centred at 30, 44 and $70 \mathrm{GHz}$. The LFI is sensitive to polarisation in all channels, a characteristic of coherent detectors that does not call for any additional component or system compromise. The $70 \mathrm{GHz}$ channel is near the minimum of the foreground emission, thus probing the cleanest cosmological window with an angular resolution of 13'. The 30 and $44 \mathrm{GHz}$ channels are sensitive to the cosmological signal but also to synchrotron, free-free, and anomalous dust diffuse radiation from the Galaxy. Thus they will serve as cosmological and foreground monitors in the Planck observations.

The LFI design required several challenging trade offs involving thermal, mechanical, electrical, and optical aspects (Table 13). The cryogenic front-end receivers, required for high sensitivity, dominate the instrument performance and their interface with HFI is a major driver of the instrument configuration. The combination of the pseudo-correlation scheme and of the $4 \mathrm{KHz}$ switching of the phase shifters in the FEM allow us to obtain excellent stability while maintaining a highly modular design. Stringent requirements on noise temperature, $1 / f$ noise, thermal and electrical stability, bandwidth, polarisation isolation, and parasitic heat loads were key elements in the design. Another key driver in the LFI design has been the control of systematic effects, which has also been a central part of the LFI calibration plan and test campaigns, both on-ground and in-flight. The functionality and performance of LFI was tested at various stages of development and integration (component level, unit level, RCA, instrument, and satellite level) and have been measured again in flight. The achieved performances based on ground testing are described by Mennella et al. (2010) and Villa et al. (2010) and are generally in line with the design expectations.

Acknowledgements. The Planck-LFI project is developed by an International Consortium led by Italy and involving Canada, Finland, Germany, Norway, Spain, Switzerland, UK, USA. The Italian contribution to Planck is supported by the Italian Space Agency (ASI). T.P.'s work was supported in part by the Academy of Finland grants 205800, 214598, 121703, and 121962. T.P. thanks the Waldemar von Frenckells Stiftelse, Magnus Ehrnrooth Foundation, and Väisälä Foundation for financial support. We acknowledge partial support from the NASA LTSA Grant NNG04CG90G. 
M. Bersanelli et al.: Planck pre-launch status: Design and description of the Low Frequency Instrument

\section{References}

Artal, E., Aja, B., L. de la Fuente, M., et al. 2009, JINST, 4, T12003 Battaglia, P., Bersanelli, M., Butler, R., et al. 2009, JINST, 4, T12014

Bersanelli, M., \& Mandolesi, N. 2000, Astroph. Lett. Commun., 37, 171

Bersanelli, M., Bouchet, F., Efstathiou, G., et al. 1996a, Report on Phase A Study of COBRAS/SAMBA, ESA publ. ESA D/SCI (96)

Bersanelli, M., Mandolesi, N., Cesarsky, C., et al. 1996b, Astrophys. Lett. Commun., 33, 19

Bhandari, P., Prina, M., Bowman, R. C., et al. 2004, Cryogenics, 44, 395

Blum, E. 1959, Ann. Astrophys., 22(2), 140

Burigana, C., Maino, D., Mandolesi, N., et al. 1998, A\&AS, 130, 551

Burigana, C., Maino, D., Górski, K. M., et al. 2001, A\&A, 373, 345

Cuttaia, F., Menella, A., Stringhetti, L., et al. 2009, JINST, 4, T12013

D’Arcangelo, O., Figini, L., Simonetto, A., et al. 2009, JINST, 4, T12007

Davis, R., Wilkinson, A., Davies, R., et al. 2009, JINST, 4, T12002

Herreros, J., Gómez, M., Rebolo, R., et al. 2009, JINST, 4, T12008

Hoyland, R. 2003, in ESA Workshop on Millimetre Wave Technology and ich Applications, Proceedings of 3rd ESA Workshop on Millimetre Wave Technology and Applications, 211, 305

Jarosik, N. 1996, IEEE Trans. on Microwave Theory and Techniques, 44, 193

Jarosik, N., Bennett, C. L., Halpern, M., et al. 2003, ApJS, 145, 413

Keihänen, E., Kurki-Suonio, H., Poutanen, T., Maino, D., \& Burigana, C. 2004, A\&A, 428, 187

Knox, L. 1995, Phys. Rev. D, 52, 4307

Lamarre, J.-M., Puget, J.-L., Ade, P. A. R., et al. 2010, A\&A, 520, A9

Leahy, J. P., Bersanelli, M., D’Arcangelo, O., et al. 2010, A\&A, 520, A8

Leutenegger, P., Bersanelli, M., Ferretti, R., \& Prina, M. 2003, in Cryogenic Optical Systems and Instruments, ed. J. Heaney, \& L. Burriesci, SPIE, 5172, 130

Maffei, B., Noviello, F., Murphy, J. A., et al. 2010, A\&A, 520, A12

Maino, D., Burigana, C., Maltoni, M., et al. 1999, A\&A, 140, 383

Maino, D., Burigana, C., Górski, K. M., Mandolesi, N., \& Bersanelli, M. 2002, A\&A, 387, 356

Mandolesi, N., Bersanelli, M., Burigana, C., et al. 2000a, A\&AS, 145, 323

Mandolesi, N., Bersanelli, M., Burigana, C., \& Villa, F. 2000b, Astrophys. Lett. Commun., 37, 151

Mandolesi, N., Bersanelli, M., Butler, R. C., et al. 2010, A\&A, 520, A3

Maris, M., Bersanelli, M., D’Arcangelo, O., et al. 2009, JINST, 4, T12018

Meinhold, P., Leonardi, R., Aja, B., et al. 2009, JINST, 4, T12009

Mennella, A., \& Bersanelli, M. 2001, Impact of Back-End temperature fluctuations on LFI PLANCK-LFI, Tech. Rep. PL-LFI-PST-TN-029, IASF-MICNR

Mennella, A., Bersanelli, M., Burigana, C., et al. 2002a, A\&A, 384, 736

Mennella, A., Seiffert, M., \& Bersanelli, M. 2002b, Temperature stability requirements of the LFI Sorption Cooler cold-end (LR2), Tech. rep., IASFMI-CNR

Mennella, A., Bersanelli, M., Seiffert, M., et al. 2003, A\&A, 410, 1089

Mennella, A., Baccigalupi, C., Balbi, A., et al. 2004, Res. Devel. Astron. \& Astrophys., 2, 1

Mennella, A., Bersanelli, M., Butler, R. C., et al. 2010, A\&A, 520, A5

Morgante, G., Pearson, D., Melot, F., et al. 2009, JINST, 4, T12016

Penzias, A. A., \& Wilson, R. W. 1965, ApJ, 142, 419

Planck Collaboration 2005, Planck: The Scientific Programme, ESA Publ. ESA-SCI (2005)/1

Sandri, M., Bersanelli, M., Mennella, A., et al. 2010, A\&A, 520, A7

Seiffert, M., Mennella, A., Burigana, C., et al. 2002, A\&A, 391, 1185

Staggs, S. T., Jarosik, N. C., Wilkinson, D. T., \& Wollack, E. J. 1996, ApJ, 458, 407

Tauber, J. A., Mandolesi, N., Puget, J.-L., et al. 2010a, A\&A, 520, A1

Tauber, J. A., Norgaard-Nielsen, H. U., Ade, P. A. R., et al. 2010b, A\&A, 520, A2

Tomasi, M., Cappellini, B., Gregorio, A., et al. 2010, JINST, 5, T01002

Valenziano, L., Cuttaia, F., De Rosa, A., et al. 2009, JINST, 4, T12006

Varis, J., Hughes, N., Laaninen, M., et al. 2009, JINST, 4, T12001

Villa, F., Sandri, M., Mandolesi, N., et al. 2002, Exp. Astron., 14, 1

Villa, F., D’Arcangelo, O., Pagana, E., et al. 2009a, JINST, 4, T12005

Villa, F., D'Arcangelo, O., Pecora, M., et al. 2009b, JINST, 4, T12004

Villa, F., Terenzi, L., Sandri, M., et al. 2010, A\&A, 520, A6

Wade, L., Bhandari, P., Bowman, J. R., et al. 2000, in Advances in Cryogenic Engineering, ed. Q.-S. Shu, et al. (New York: Kluwer Academic/Plenum), 45A, 499
Zacchei, A., Frailis, M., Maris, M., et al. 2009, JINST, 4, T12019

Zonca, A., Franceschet, C., Battaglia, P., et al. 2009, JINST, 4, T12010

1 Università degli Studi di Milano, Dipartimento di Fisica, via Celoria 16, 20133 Milano, Italy e-mail: marco.bersanelli@unimi.it

2 INAF - Istituto di Astrofisica Spaziale e Fisica Cosmica, via Bassini 15, 20133 Milano, Italy

3 INAF - Istituto di Astrofisica Spaziale e Fisica Cosmica, via P. Gobetti, 101, 40129 Bologna, Italy

${ }^{4}$ Universidad de Cantabria, Departamento de Ingenieria de Comunicaciones, Av. de Los Castros s/n, 39005 Santander, Spain

5 Thales Alenia Space Italia S.p.A., S.S. Padana Superiore 290, 20090 Vimodrone, Milano, Italy

6 SISSA/ISAS, Astrophysics Sector, Via Beirut 4, 34014 Trieste, Italy

7 CESR, Centre d'Étude Spatiale des Rayonnements, 9 Av. du Colonel Roche, BP 44346, 31028 Toulouse Cedex 4, France

8 Instituto de Fisica de Cantabria, CSIC, Universidad de Cantabria, Av. de los Castros s/n, 39005 Santander, Spain

9 Jodrell Bank Centre for Astrophysics, Alan Turing Building, The University of Manchester, Manchester, M13 9PL, UK

10 Herschel/Planck Project, Scientific Projects Dpt of ESA, Keplerlaan 1, 2200 AG, Noordwijk, The Netherlands

11 Istituto di Fisica del Plasma, CNR, via Cozzi 53, 20125 Milano, Italy

12 ASI, Agenzia Spaziale Italiana, viale Liegi, 26, 00198 Roma, Italy

13 Dipartimento di Fisica, Università degli Studi di Roma Tor Vergata, via della Ricerca Scientifica 1, 00133 Roma, Italy

14 INAF - Osservatorio Astronomico di Padova, Vicolo dell'Osservatorio 5, 35122 Padova, Italy

15 INAF - Osservatorio Astronomico di Trieste, via Tiepolo, 11, 34143 Trieste, Italy

16 Jet Propulsion Laboratory, California Institute of Technology, 4800 Oak Grove Drive, Pasadena, CA 91109, USA

17 Instituto de Astrofisica de Canarias, C/ via Lactea s/n, 38200 La Laguna, Tenerife, Spain

18 Dipartimento di Fisica, Università degli Studi di Trieste, via A. Valerio 2, 34127 Trieste, Italy

19 DA-Design Oy, Keskuskatu 29, 31600 Jokioinen, Finland

20 Ylinen Electronics Oy, Teollisuustie 9A, 02700 Kauniainen, Finland

21 Department of Physics, University of California, Santa Barbara, CA 93106, USA

22 Institute of Theoretical Astrophysics, University of Oslo, PO Box 1029 Blindern, 0315 Oslo, Norway

23 INAF - Osservatorio Astrofisico di Arcetri, Largo Enrico Fermi 5, 50125 Firenze, Italy

24 Haverford College, 370 Lancaster Avenue, Haverford, PA 19041, USA

25 National Radio Astronomy Observatory, 520 Edgemont Rd, Charlottesville, VA 22903-2475, USA

26 University of Helsinki, Department of Physics, PO Box 64, 00014 Helsinki, Finland

27 Helsinki Institute of Physics, University of Helsinki, PO Box 64, 00014, Finland

28 Metsähovi Radio Observatory, Helsinki University of Technology, Metsähovintie 114, 02540, Kylmälä, Finland

29 Lawrence Berkeley National Laboratory, 1 Cyclotron Road, Berkeley, CA 94720, USA

30 European Space Agency (ESA), Astrophysics Division, Keplerlaan 1, 2201AZ Noordwijk, The Netherlands

31 MilliLab, VTT Technical Research Centre of Finland, PO Box 1000, 02044 VTT, Finland

32 MPA Max-Planck-Institut für Astrophysik, Karl-Schwarzschild-Str. 1, 85741 Garching, Germany 